\title{
A Decision Variable Clustering-Based Evolutionary Algorithm for Large-scale Many-objective Optimization
}

\author{
Xingyi Zhang, Ye Tian, Ran Cheng and Yaochu Jin, Fellow, IEEE
}

\begin{abstract}
The current literature of evolutionary manyobjective optimization is merely focused on the scalability to the number of objectives, while little work has considered the scalability to the number of decision variables. Nevertheless, many real-world problems can involve both many objectives and large-scale decision variables. To tackle such large-scale many-objective optimization problems, this paper proposes a specially tailored evolutionary algorithm based on a decision variable clustering method. To begin with, the decision variable clustering method divides the decision variables into two types: convergence-related variables and diversity-related variables. Afterwards, to optimize the two types of decision variables, a convergence optimization strategy and a diversity optimization strategy are adopted. In addition, a fast non-dominated sorting approach is developed to further improve the computational efficiency of the proposed algorithm. To assess the performance of the proposed algorithm, empirical experiments have been conducted on a variety of large-scale many-objective optimization problems with up to 10 objectives and 5000 decision variables. Our experimental results demonstrate that the proposed algorithm has significant advantages over several state-of-the-art evolutionary algorithms in terms of the scalability to decision variables on many-objective optimization problems.
\end{abstract}

Index Terms-Evolutionary multi-objective optimization, many-objective optimization, large-scale optimization, clustering, non-dominated sorting, tree

\section{INTRODUCTION}

$\mathbf{M}$ ANY-objective optimization problems (MaOPs) refer to the problems that involve more than three conflicting objectives to be optimized simultaneously, which widely exist in real-world applications, such as engineering design [1], air traffic control [2], groundwater monitoring [3] and molecular design [4]. Generally

Manuscript received -. This work was supported in part by National Natural Science Foundation of China (Grant No. 61272152, 61502004, 61502001), and the Joint Research Fund for Overseas Chinese, Hong Kong and Macao Scholars of the National Natural Science Foundation of China (Grant No. 61428302). (Corresponding author: Ran Cheng)

Xingyi Zhang and Ye Tian are with the Institute of Bio-inspired Intelligence and Mining Knowledge, School of Computer Science and Technology, Anhui University, Hefei 230039, China (email: xyzhanghust@gmail.com; field910921@gmail.com).

Ran Cheng is with the School of Computer Science, University of Birmingham, Birmingham, B15 2TT, United Kingdom (email: ranchengcn@gmail.com).

Yaochu Jin is with the Department of Computer Science, University of Surrey, Guildford, Surrey, GU2 7XH, United Kingdom (email: yaochu.jin@surrey.ac.uk). speaking, MaOPs can not be easily solved by most multiobjective evolutionary algorithms (MOEAs) designed for dealing with traditional multi-objective optimization problems (MOPs) which involve only two or three objectives due to two major issues [5]-[9]. The first issue is the loss of convergence pressure, which is mainly caused by the phenomenon called dominance resistance [10]. This is due to the fact that in many-objective optimization, most candidate solutions become non-dominated with each other, thus causing failure of dominance based selection strategies in traditional MOEAs. The other issue is the difficulty in diversity management. In many-objective optimization, the candidate solutions are sparsely distributed in the high-dimensional objective space, which leads to the ineffectiveness of traditional diversity management strategies. To tackle the above two issues, a number of approaches have been proposed [11], [12], which can be roughly divided into four categories.

The first category covers various convergence enhancement based approaches. To enhance the convergence pressure, the most intuitive idea is to directly modify the definition of traditional Pareto dominance, such as $\varepsilon$-dominance [13], [14], $L$-optimality [15], fuzzy dominance [16], preference order ranking [17] and $\theta$ dominance [18]. Another idea belonging to this category is to combine the traditional dominance with an additional convergence-related metric, and MOEAs belonging to this category include the substitute distance assignment based NSGA-II [19], grid-based evolutionary algorithm (GrEA) [20], preference-inspired coevolutionary algorithm (PICEA-g) [21], many-objective evolutionary algorithm based on directional diversity and favorable convergence (MaOEA-DDFC) [22], and knee point driven evolutionary algorithm (KnEA) [23].

The second category directly adopts performance indicators as selection criterion to distinguish nondominated solutions which can not be distinguished by traditional Pareto dominance. Among many others [24]-[27], some representative approaches of this catetory are indicator based evolutionary algorithm (IBEA) [28], $S$-metric selection based evolutionary multiobjective optimization algorithm (SMS-EMOA) [29] and hypervolume-based evolutionary algorithm (HypE) [30].

The third category is known as the decomposition 
based approaches, where the idea is to decompose an $\mathrm{MaOP}$ into a group of simple sub-problems and then solve them in a collaborative manner. To be specific, one type of decomposition is to decompose an MaOP into a group of single-objective problems (SOPs). One popular decomposition based approach of this type is the MOEA/D algorithm [31], as well as its variants, such as stable matching model based MOEA/D (MOEA/DSTM) [32], MOEA/D with adaptive weight vector adjustment (MOEA/D-AWA) [33], external archive guided MOEA/D (EAG-MOEA/D) [34], MOEA/D with a distance based updating (MOEA/D-DU) [35] and online diversity metric based MOEA/D [36]. Another type of decomposition is to decompose an MaOP into a group of simple MOPs. Some representative MOEAs of this type are MOEA/D-M2M [37], reference-point based manyobjective NSGA-II (NSGA-III) [38], dominance and decomposition based MOEA (MOEA/DD) [39], and the recently proposed reference vector guided evolutionary algorithm (RVEA) [40].

The main idea of the fourth category is to convert an MaOP into an MOP, so that it can be directly solved using existing MOEAs. There are two major branches along this direction. One branch is to use the objective reduction approaches to eliminate redundant or irrelevant objectives, such as dominance relation preservation based algorithms [41], [42], unsupervised feature selection based algorithms [43], Pareto corner search based algorithms [44], machine learning based objective reduction [45]-[47], and the recently proposed nonlinear correlation information entropy based objective reduction [48]. The other branch is to replace the original objectives with two or three newly defined objectives. Representative approaches of this type include bi-goal evolution (BiGE) [49] and summation of normalized objective values and diversified selection based MOEA (SNOV-DS) [50].

Apart from the approaches proposed to enhance the search abilities of MOEAs in many-objective optimization, there is also some work motivated to improve the computational efficiency. For example, Bringmann et al. suggested to use the Monte Carlo method to improve the computational efficiency of hypervolume calculation$\mathrm{s}$ in the multi-objective covariance matrix adaptation evolution strategy (MO-CMA-ES) [51]. Some fast nondominated sorting approaches were also developed to reduce the computational cost of Pareto-based MOEAs for solving MaOPs, such as deductive sort [52], corner sort [53], M-front [54], efficient non-dominated sort (ENS) [55] and approximate non-dominated sort (AENS) [56].

However, in spite of the various approaches that are focused on the scalability of MOEAs to the number of objectives, little work has been reported to consider the large number of decision variables in multi-objective optimization. While large-scale optimization has already attracted certain interests in the single-objective literature [57]-[63], the development of large-scale multi- objective optimization is still in the infancy [64].

Recently, Ma et al. have proposed a decision variable analysis based MOEA, known as MOEA/DVA, for solving large-scale MOPs [65]. In MOEA/DVA, a decision variable analysis method based on dominance relationships is designed to divide the decision variables into three groups: convergence-related variables, diversity-related variables and variables related to both convergence and diversity. The convergence-related variables and diversity-related variables are optimized with different strategies separately, while the variables related to both convergence and diversity are simply treated as diversity-related variables. It has been shown that such a decision variable analysis method works efficiently on large-scale MOPs with two or three objectives, although its performance has not yet been assessed on any MaOP.

Following the basic idea of MOEA/DVA proposed in [65], in this work, we propose an evolutionary algorithm for tackling large-scale MaOPs based on a decision variable clustering method, where the main new contributions are summarized as follows.

1) A decision variable clustering method is proposed to divide the decision variables into convergencerelated and diversity-related ones. While the decision variable analysis method in MOEA/DVA [65] is based on dominance based relationships, the proposed decision variable clustering method adopts the $k$-means method with features measured by the angles between the sample solutions and the direction of convergence, where smaller angles indicate more contributions to convergence and larger angles to diversity. Consequently, a decision variable can only be divided as either convergence-related or diversity-related, thus addressing the open issue that decision variables related to both convergence and diversity can not be further distinguished [65].

2) An evolutionary algorithm for large-scale manyobjective optimization (LMEA) is proposed based on the decision variable clustering method. In LMEA, two optimization strategies, i.e., the convergence optimization strategy and the diversity optimization strategy, are proposed to deal with the two types of decision variables separately. Both strategies adopt non-dominated sorting as the first selection criterion but differ in the secondary selection criterion: to enhance convergence, the secondary selection in the convergence optimization strategy is based on the Euclidean distances from the candidate solutions to the ideal point; to manage diversity, the secondary selection in the diversity optimization strategy is based on the angles between the candidate solutions.

3) To reduce the computational cost of the two optimization strategies in LMEA, a computational efficient tree-based non-dominated sorting approach, termed T-ENS, is developed on the basis of the recently proposed ENS [55]. In T-ENS, the information for identifying the non-dominance relationship 
is recorded in the nodes of a tree, with which a large number of non-dominance comparisons between solutions can be inferred. As a result, only a sub-set of the solutions, rather than all, that have been assigned to a non-dominated front needs to be compared. Theoretical analysis indicates that the developed T-ENS holds a time complexity of $O(M N \log N / \log M)$, where $N$ denotes the population size and $M$ denotes the number of objectives, which is more efficient than the average complexity of $O\left(M N^{2}\right)$ held by most existing approaches for solving MaOPs where most solutions in the populations are non-dominated with each other.

4) To assess the performance of the proposed LMEA, empirical evaluations have been conducted on a variety of benchmark problems in comparison with several state-of-the-art MOEAs for solving MaOPs as well as large-scale MOPs. Our experimental results demonstrate that LMEA is well suited for solving large-scale MaOPs having up to 5000 decision variables.

The rest of the paper is organized as follows. In Section II, we briefly recall some related work on MOEAs for solving large-scale MOPs. The motivation of this work is also elaborated in Section II, mainly with respect to the recently proposed MOEA/DVA. In Section III, we describe the details of the proposed LMEA for large-scale MaOPs. Simulation results are presented in Section IV to empirically assess performance of the LMEA on largescale MaOPs. Finally, conclusions and future work are drawn in Section V.

\section{RELATED WORK AND MOTIVATION}

In this section, we first review several representative MOEAs for solving large-scale MOPs, and then elaborate the motivation of this work by two illustrative examples. Note that, the current literature only covers a very limited number of MOEAs that can achieve a good performance on large-scale MOPs, some of which were even not explicitly designed for solving large-scale MOPs.

Antonio and Coello suggested a cooperative coevolution framework for solving large-scale MOPs in [64]. The main idea of this algorithm is to randomly divide the large number of decision variables into several small subcomponents of equal size and coevolve these subcomponents cooperatively for a predefined number of cycles. Experimental results confirmed the competitiveness of the algorithm on large-scale MOPs with two and three objectives. Following this line, in [66], the idea of cooperative coevolution was also adopted to deal with large-scale multi-objective capacitated arc routing problems.

Most recently, an MOEA for solving large-scale MOPs, termed MOEA/DVA, has been suggested by Ma et al. in [65], where a decision variable analysis strategy is adopted to divide the decision variables into different

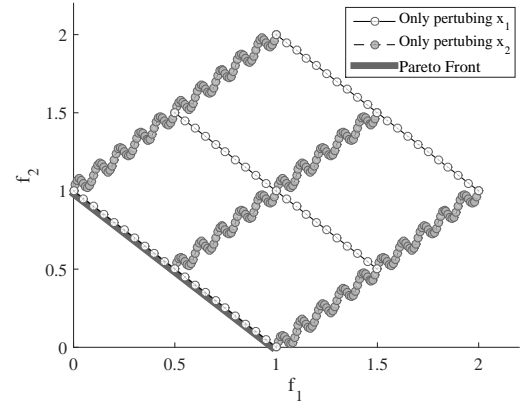

Fig. 1. The sampling points obtained on the MOP formulated in (1) by perturbing one variable and fixing the other to $0,0.5$ and 1 , respectively.

groups by checking the dominance relationships between solutions generated by perturbing the values of variables. To be specific, a decision variable is considered to be:

1) convergence-related, if and only if the solutions generated by the perturbed decision variable values are dominated one by one;

2) diversity-related, if and only if the solutions generated by the perturbed decision variable values are non-dominated with each other;

3) related to both convergence and diversity, otherwise.

Once the decision variables are successfully divided into different groups, MOEA/DVA performs a two-stage optimization process: firstly, the convergence-related variables are optimized until the candidate solutions reach the Pareto front; afterwards, the diversity-related variables are optimized to achieve a wide distribution of the candidate solutions on the Pareto front.

For the variables related to both convergence and diversity, MOEA/DVA simply treats them as diversityrelated variables, as the variable analysis strategy is not able to further distinguish them [65]. Nevertheless, our empirical observation indicates that, these decision variables still can be further divided into two groups, as some of them contribute more to convergence while others contribute more to diversity. To illustrate such an observation, let us consider the following bi-objective MOP:

$$
\left\{\begin{array}{l}
\min f_{1}=x_{1}+x_{2} \\
\min f_{2}=\left(1-x_{1}\right)+x_{2}+\sin \left(20 \pi x_{2}\right) / 20 \\
\text { subject to: } x_{i} \in[0,1], i=1,2
\end{array}\right.
$$

Fig. 1 shows the sampling points obtained on the MOP formulated in (1) by perturbing one variable between $[0,1]$ while fixing the other to $0,0.5$ and 1 , respectively. According to the variable analysis strategy introduced above, MOEA/DVA labels $x_{1}$ and $x_{2}$ as a diversityrelated variable and a variable related to both convergence and diversity, respectively. Nevertheless, since $x_{2}$ contributes more to convergence than diversity, it will be more beneficial to optimize $x_{2}$ as a convergence-related variable rather than a diversity-related one.

It should be noted that, even for certain diversityrelated variables, sometime it is also essential to consider 


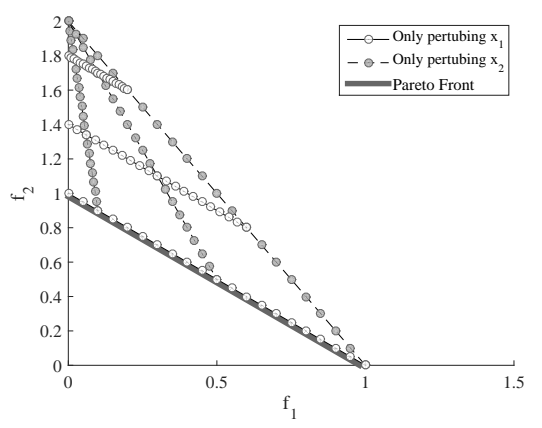

Fig. 2. The sampling points obtained on the MOP formulated in (2) by perturbing one variable between $[0,1]$ while fixing the other as 0.2 , 0.6 and 1 , respectively.

them as convergence-related to guarantee convergence of the population. Let us take the following bi-objective MOP as an example:

$$
\left\{\begin{array}{l}
\min f_{1}=x_{1} x_{2} \\
\min f_{2}=\left(1-x_{1} x_{2}\right)+1-x_{2} \\
\text { subject to: } x_{i} \in[0,1], i=1,2
\end{array}\right.
$$

Fig. 2 shows the sampling points obtained on the MOP formulated in (2) by perturbing one variable between $[0,1]$ while fixing the other to $0.2,0.6$ and 1 , respectively. According to the variable analysis strategy introduced above, MOEA/DVA labels both $x_{1}$ and $x_{2}$ as diversityrelated variables. Nevertheless, in this case, it is more beneficial to label $x_{2}$ as a convergence-related variable due to the fact that optimizing $x_{2}$ will guide the population towards the Pareto front.

To address the above issue, this paper suggests a decision variable clustering based MOEA, termed LMEA, for large-scale MaOPs. In LMEA, a decision variable clustering method is proposed to distinguish convergencerelated and diversity-related variables by measuring their contributions to convergence and diversity, respectively. With the proposed decision variable clustering method, variables similar to $x_{2}$ in the above two examples can be properly categorized.

\section{The Proposed Algorithm: LMEA}

In this section, we first present the main framework of the proposed LMEA, then detail two important components in it, i.e., the decision variable clustering method and the fast tree-based non-dominated sorting approach.

\section{A. The Main Framework of LMEA}

Algorithm 1 presents the main framework of LMEA, which consists of the following five components. First, a population of $N$ candidate solutions is randomly initialized. Second, the developed decision variable clustering method is applied to divide the variables into two group$\mathrm{s}$, convergence-related variables and diversity-related variables. Third, the convergence-related variables are further divided into several subgroups based on the interaction between these variables, where the variables are interacted with each other inside one subgroup but

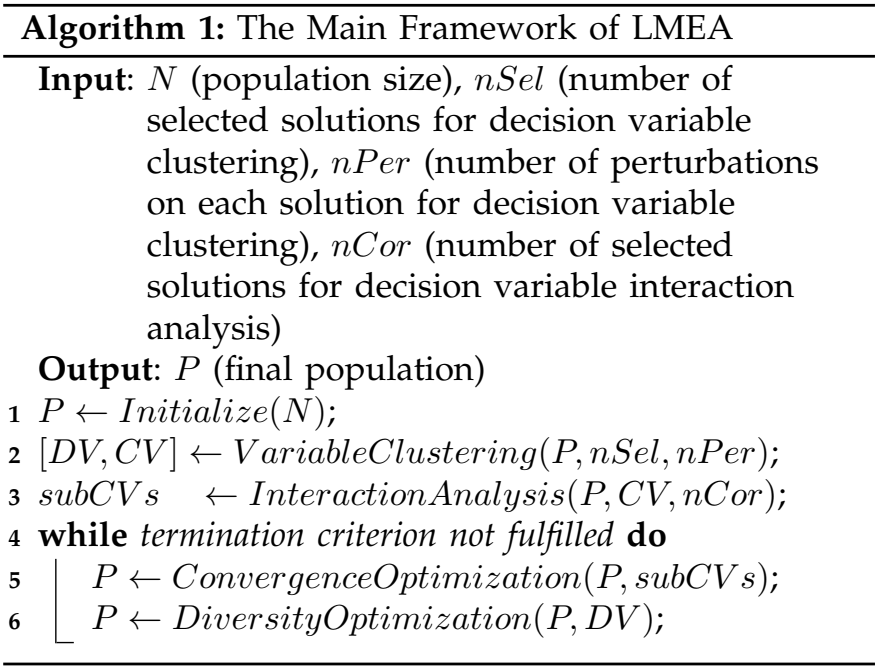

not interacted with those in any other subgroups. The variables in each subgroup are also known as interacting variables, as they can not be optimized separately due to the interactions between each other. Algorith$\mathrm{m} 2$ presents the details of the interaction analysis for convergence-related variables, which adopts the strategy developed in [65]. Note that similar strategies have also been widely used for variable interaction detection in single-objective large-scale optimization [57], [67].

Algorithm 2 first initializes an empty set $s u b C V s$ of interacted variable subgroups, and afterwards, the convergence-related variables in $C V$ are assigned to different subgroups based on the pairwise interactions between them, where the interactions are defined as follows: given an MOP $\min \mathbf{f}=\min \left(f_{1}, f_{2}, \ldots, f_{m}\right)$, if there exist $\mathbf{x}, a_{1}, a_{2}, b_{1}, b_{2}$ and at least one $f_{k}$, $1 \leq k \leq m$, such that $\left.f_{k}(\mathbf{x})\right|_{x_{i}=a_{2}, x_{j}=b_{1}}<\left.f_{k}(\mathbf{x})\right|_{x_{i}=a_{1}, x_{j}=b_{1}}$ and $\left.f_{k}(\mathbf{x})\right|_{x_{i}=a_{2}, x_{j}=b_{2}}>\left.f_{k}(\mathbf{x})\right|_{x_{i}=a_{1}, x_{j}=b_{2}}$, then the two decision variables $x_{i}$ and $x_{j}$ are regarded to be interacted, where $\left.f_{k}(\mathbf{x})\right|_{x_{i}=a_{2}, x_{j}=b_{1}}=$ $f_{k}\left(x_{1}, \ldots, x_{i-1}, a_{2}, \ldots, x_{j-1}, b_{1}, \ldots, x_{n}\right)$. To be specific, if a variable has interaction with at least one existing variable in $s u b C V s$, then the two variables are assigned into the same subgroup; otherwise, it is assigned to a new subgroup. This operation is repeated until each convergence-related variable has been assigned to a subgroup. Therefore, in the extreme cases, there will be at most a number of $|C V|$ subgroups, which means that the convergence-related variables are fully separable; while if the variables are fully non-separable, there will be only one subgroup.

Once the interaction analysis is done in Algorithm 2, LMEA starts to optimize each subgroup of variables one by one using a convergence optimization strategy as detailed in Algorithm 3, while the diversity-related variables are optimized using a diversity optimization strategy as detailed in Algorithm 4.

In the convergence optimization strategy, the proposed T-ENS is used to perform non-dominated sorting on the parent population, and the Euclidean distance from 


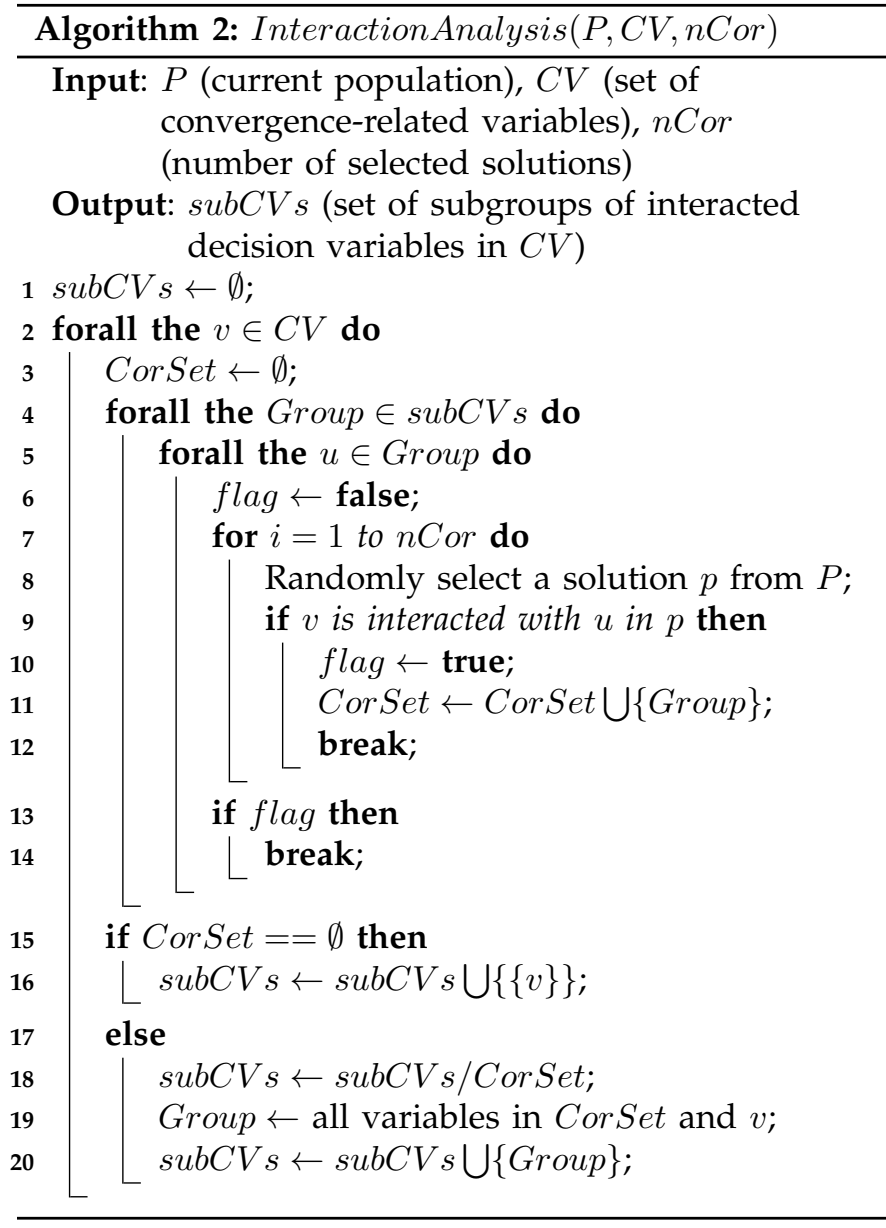

each candidate solution to the ideal point ${ }^{1}$ is calculated. Afterwards, the offspring candidate solutions are generated by optimizing the convergence-related variables inside each subgroup independently. For the variables in each subgroup, two candidate solutions are randomly chosen from population $P$ using the binary tournament, and afterwards, an offspring candidate solution is generated by replacing the values of the variables in the same subgroup with those generated by recombination operators, while leaving the rest variables unchanged. If an offspring candidate solution has a better convergence than its parent, it will be selected to be passed to the next generation; otherwise, the offspring candidate solution is discarded and the parent candidate solution will survive. To be specific, an offspring candidate solution is considered to have better convergence quality than its parent solution if and only if it has a smaller nondominate front number in the non-dominated sorting, or it has the same non-dominated front number but a smaller Euclidean distance to the ideal point.

In the diversity optimization strategy, $|P|$ offsprings are first generated from population $P$ by optimizing all diversity-related variables as a whole using the recombination operators, then the offspring population is com-

\footnotetext{
${ }^{1}$ In this work, the candidate solutions are all transformed to be inside the first quadrant of the objective space, such that the ideal point is always the axis origin.
}

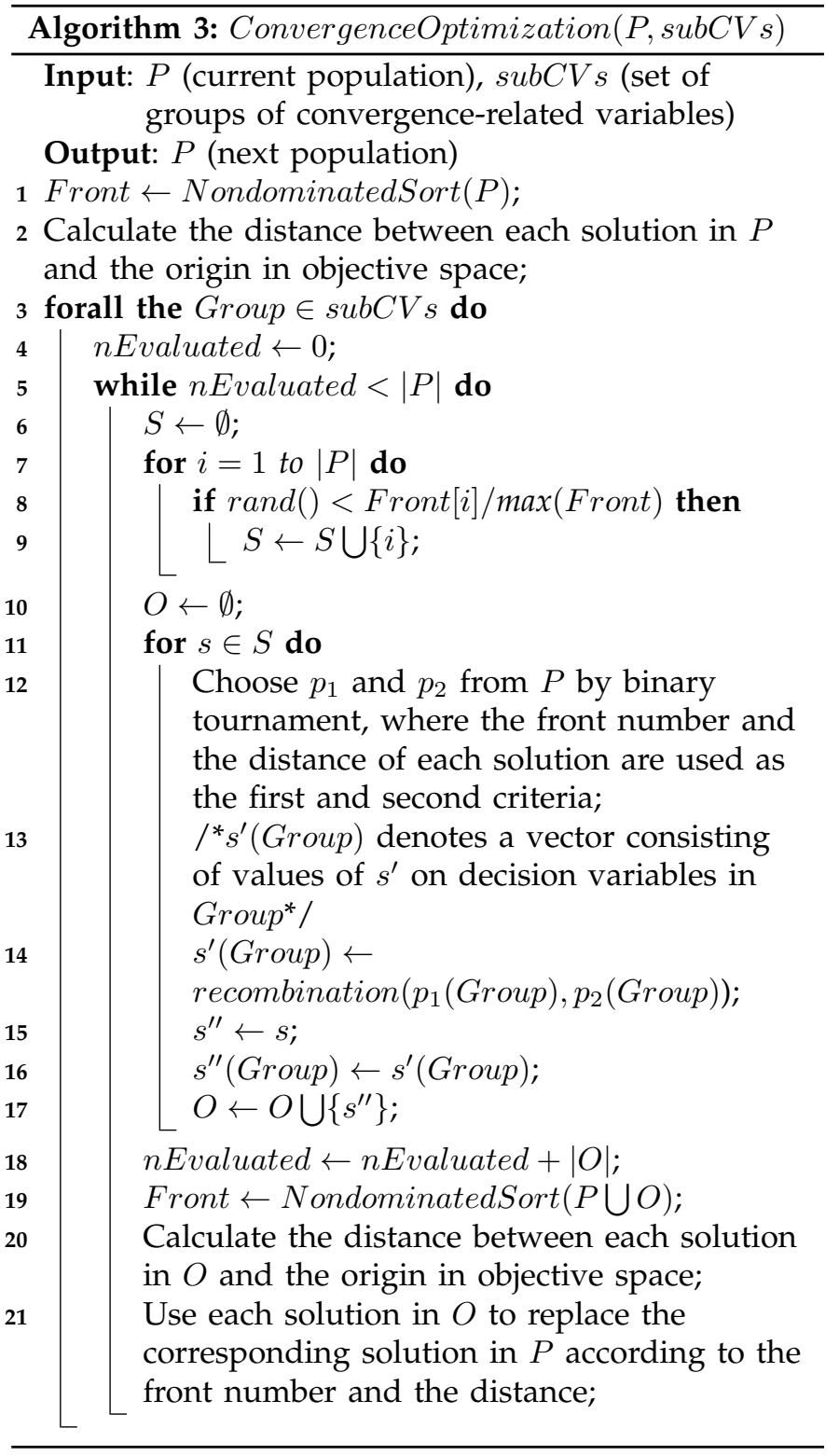

bined with the parent population and the environmental selection performed on the combined population consist$s$ of two stages: to begin with, the candidate solutions from the first $k-1$ fronts are selected, such that $k$ is the smallest value satisfying $\left|F_{1} \bigcup F_{2} \bigcup \ldots \bigcup F_{k}\right|>|P|$; afterwards, the remaining candidate solutions of the best diversity are selected one by one from front $F_{k}$ until the population size $|P|$ is reached, where the diversity is measured by the angles between the candidate solutions in the objective space. As reported by Cheng et al. in [40], angle is a more efficient diversity measurement compared to Euclidean distance in many-objective optimization. Algorithm 3 and Algorithm 4 are repeated until a termination condition is met.

It should be noted that, in both optimization strategies, any commonly adopted recombination operators such as simulated binary crossover [68], polynomial mutation [69] and differential evolution [22], [70], [71] can be used to generate offspring candidate solutions. 


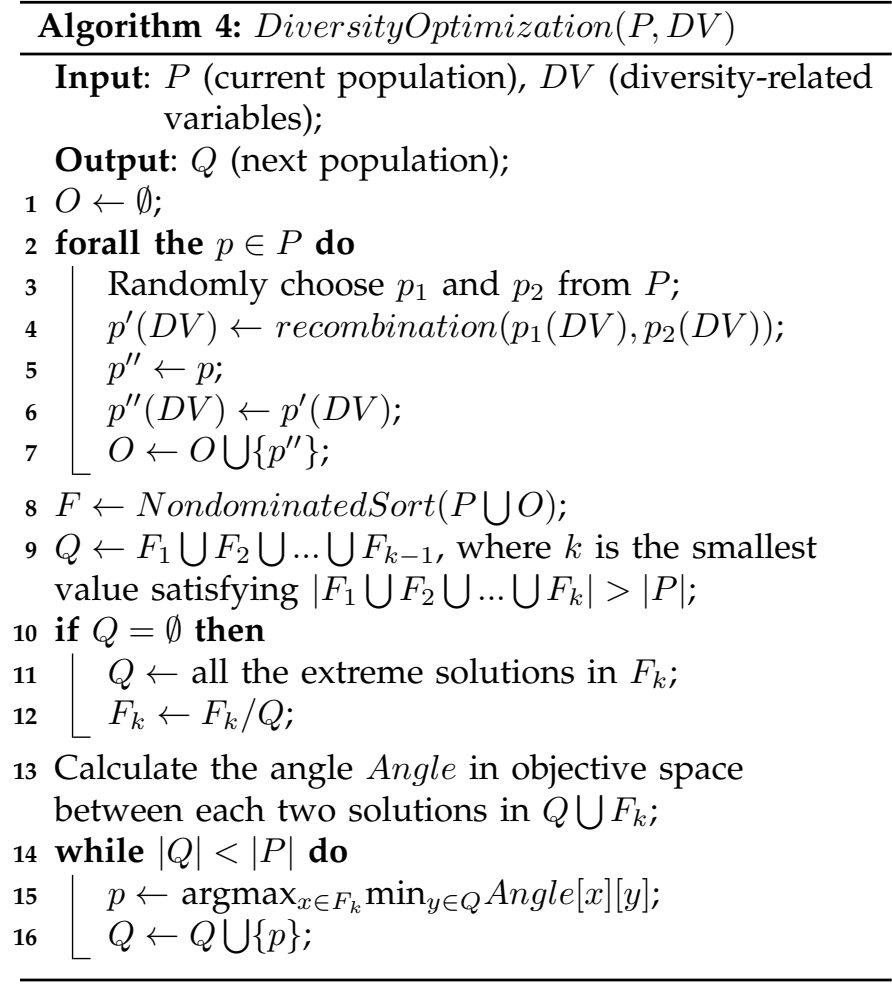

In the following subsections, we will describe in detail the decision variable clustering method and fast treebased non-dominated sorting approach, which are two most important components in the proposed LMEA.

\section{B. Decision Variable Clustering Method}

Fig. 3 presents an example to illustrate the main idea of the proposed decision variable clustering method, where a bi-objective minimization problem with four decision variables $x_{1}, x_{2}, x_{3}$ and $x_{4}$ is considered. To identify if the decision variables are convergence-related or diversity-related, a number of $n \mathrm{Sel}$ (two in this example) candidate solutions are first randomly selected from the population. Then, a number of $n P e r$ (eight in this example) perturbations are performed on each of the four variables of the selected candidate solutions. Fig. 3 (a) plots the objective values of sample solutions generated by the perturbations.

Afterwards, the sample solutions generated by perturbing the values of each variable are normalized, and a line $L$ is generated to fit each set of normalized sample solutions. With the normalized sample solutions and the fitted line $L$, the angle between each fitted line $L$ and the normal line of hyperplane $f_{1}+\ldots+f_{M}=1$ is calculated, where the normal line represents the direction of convergence and $M$ is the number of objectives. In this way, each variable is associated with several angles, where the number is dependent on the number of selected candidate solutions for the decision variable clustering. Fig. 3 (b) depicts the angles between the fitted lines and the normal line of the hyperplane. Since two candidate solutions are used for decision variable clustering in the

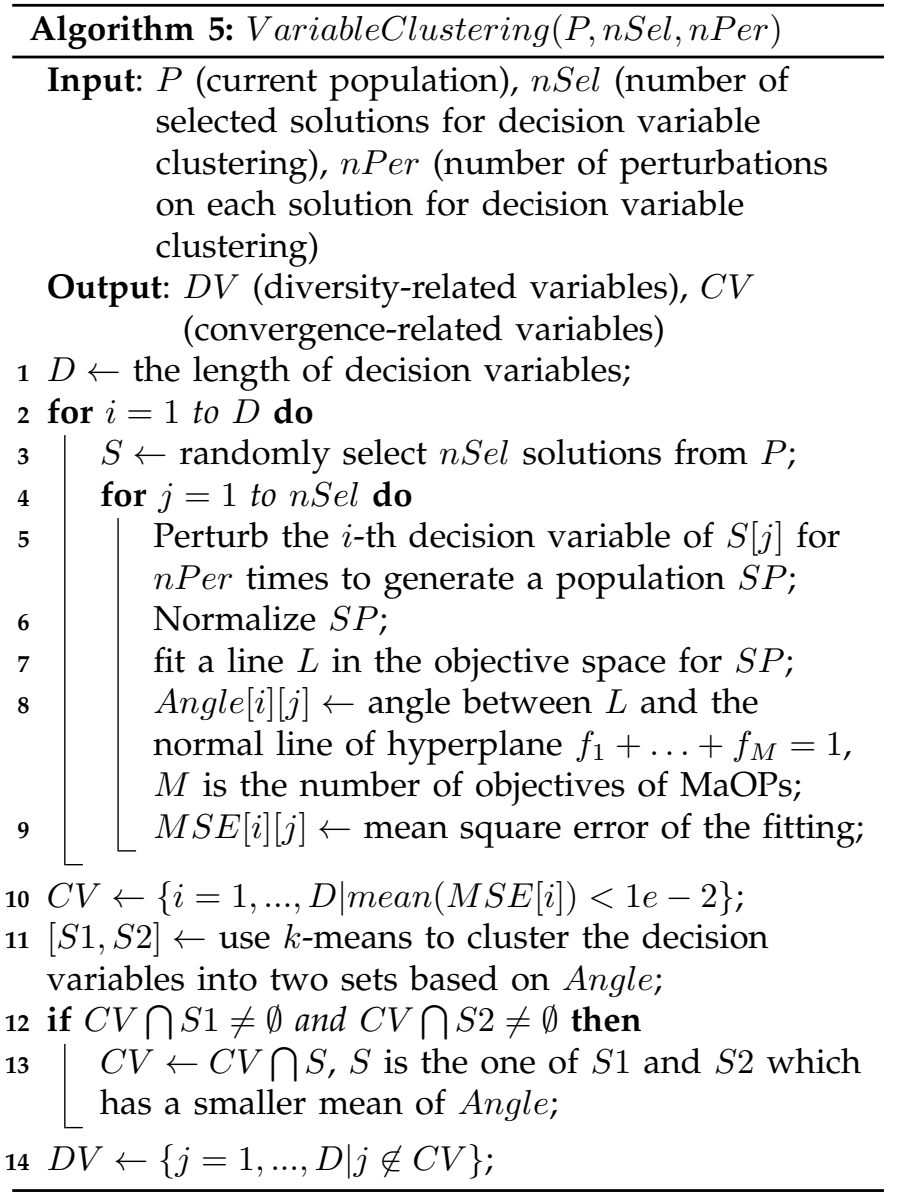

example, each variable $x_{i}, 1 \leq i \leq 4$, is associated with two angles, denoted as $\theta_{i 1}$ and $\theta_{i 2}$ as shown in Fig. 3 (b). In the proposed decision variable clustering method, the angles associated with each variable are measurements that indicate its contributions to convergence and diversity. To be specific, a larger angle means that the associated variable contributes more to diversity, while a smaller angle indicates more contribution to convergence. The more solutions are used for decision variable clustering, the more angles are associated with each variable, thus providing more precise measurements.

Finally, the $k$-means clustering method is adopted to divide the decision variables into two clusters based on the features of each variable. As a consequence, the variables in the cluster having a smaller mean of angles are identified as convergence-related, while those in the other cluster are identified as diversity-related. Fig. 3 (c) presents the clustering results on the four decision variables $x_{1}, x_{2}, x_{3}$ and $x_{4}$. As a consequence, $x_{1}$ and $x_{2}$ are identified as diversity-related variables whereas $x_{3}$ and $x_{4}$ are identified as convergence-related variables, which is consistent with the observations in Fig. 3 (a). Algorithm 5 presents the detailed procedure of the proposed decision variable clustering method.

It should be noted that, compared to the variable analysis method suggested in MOEA/DVA [65], the decision variable clustering method proposed in this work is significantly different in the following two aspects: 


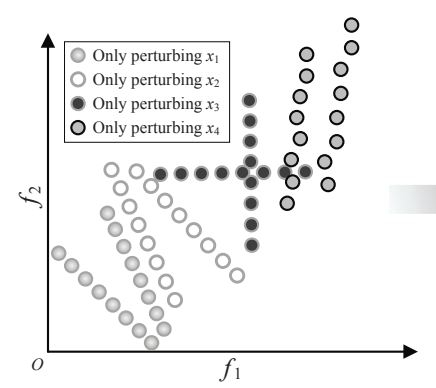

(a)

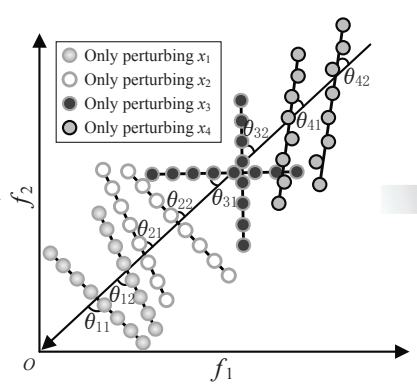

(b)

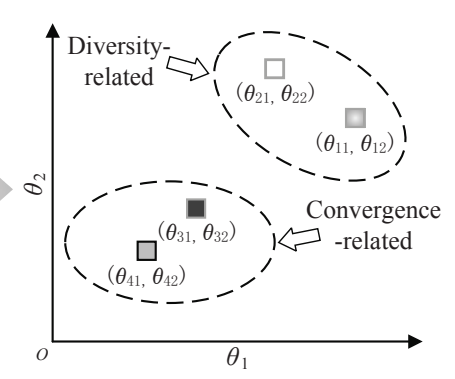

(c)

Fig. 3. An example to illustrate how to identify the convergence-related variables and diversity-related variables in LMEA for a bi-objective minimization problem with four decision variables, denoted as $x_{1}, x_{2}, x_{3}$ and $x_{4}$. In this example, $x_{1}$ and $x_{2}$ are identified as diversity-related variables, and $x_{3}$ and $x_{4}$ are identified as convergence-related variables in LMEA.

1) The decision variable analysis method in MOEA/DVA divides the variables into three categories, namely, convergence-related variables, diversity-related variables, and variables related to both convergence and diversity. By contrast, the decision variable clustering method proposed in this work divides the variables into only two categories, namely, convergence-related variables and diversity-related variables. With the proposed strategy, on the one hand, the variables identified as related to both convergence and diversity in MOEA/DVA are identified as either convergencerelated or diversity-related in LMEA; on the other hand, part of the variables identified as diversityrelated in MOEA/DVA are also identified as convergence-related in LMEA, as illustrated in Fig. 2.

2) The decision variable analysis method in MOEA/DVA determines the category of each variable based on the dominance relationship. By contrast, the proposed decision variable clustering method adopts the $k$-means method to determine the category of each variable. Compared to the decision variable analysis method in MOEA/DVA, the proposed decision variable clustering method is more robust as its performance is independent of the dominance based relationships which may probably be ineffective in many-objective optimization due to the issue of dominance resistance.

Table I presents the decision variable grouping results obtained by the decision variable analysis method in MOEA/DVA and the decision variable clustering method in LMEA on DTLZ1 to DTLZ7, WFG3, UF9 and UF10, where 16 decision variables are considered for each 5-objective DTLZ and WFG problem and all UF problems, and 15 decision variables for each 10-objective DTLZ and WFG problem. For the UF and WFG test suites, here we only list the grouping results on three representative test problems, i.e., UF9, UF10 and WFG3. The grouping results on the other WFG test problems are similar to those obtained on DTLZ1 to DTLZ4 and DTLZ7, and the results obtained on all UF problems are the same. As is evidenced in Table I, the proposed decision variable clustering method is able to further divide the variables related to both convergence and diversity as labeled by the decision variable analysis method in MOEA/DVA into convergence-related variables and diversity-related variables, respectively. In Section IV, we will empirically illustrate that the proposed decision variable clustering method in LMEA helps improve the performance of MOEAs for solving large-scale MaOPs. It is worth noting that, in practice, a decision variable can be convergence-related in a region but diversity-related in another, and vice versa. In LMEA, the convergence and diversity property of a decision variable is identified based on the perturbation results on a number of $n S e l$ solutions randomly selected from the initial population. The larger value of $n \mathrm{Sel}$ is used, the more regions will be likely to be sampled, but it does not fully guarantee the global consistency of the identification results.

\section{Fast Tree-based Non-dominated Sorting Approach}

As illustrated in Algorithms 3 and 4, non-dominated sorting is adopted during the optimization of both convergence-related variables and diversity-related variables in LMEA. Therefore, the computational efficiency of LMEA is considerably influenced by non-dominated sorting approach thus applied. In multi-objective optimization literature, there are a number of approaches that have been suggested for reducing the complexity of non-dominated sorting [52]-[55], [72]. Most of these approaches, however, are not applicable to MaOPs because they hold an average complexity of $O\left(M N^{2}\right)(M$ is the number of objectives and $N$ is the population size) if the majority of candidate solutions in a population are non-dominated with each other, which is very common in many-objective optimization though.

To reduce the computational cost of LMEA for solving large-scale MaOPs, we present a computational efficien$t$ tree-based non-dominated sorting approach, termed T-ENS, on the basis of the ENS recently proposed in [55]. The proposed T-ENS holds a time complexity of $O(M N \log N / \log M)$ for populations where most candidate solutions are non-dominated with each other. 
TABLE I

Decision Variable Grouping Results Obtained by the Decision Variable Analysis Method in MOEA/DVA and the Decision VARIAble Clustering MeThod in LMEA ON DTLZ1 to DTLZ7, WFG3, UF9 AND UF10.

\begin{tabular}{|c|c|c|c|c|c|c|c|}
\hline \multirow{2}{*}{ Problem } & \multirow{2}{*}{ Obj. } & \multicolumn{3}{|c|}{ MOEA/DVA } & \multicolumn{2}{|c|}{ LMEA } & \multirow{2}{*}{ Remark } \\
\hline & & Diversity & Convergence & Both & Diversity & Convergence & \\
\hline \multirow{2}{*}{ DTLZ1 } & 5 & $\left\{x_{1}, x_{2}, x_{3}, x_{4}\right\}$ & $\left\{x_{5}, \ldots, x_{16}\right\}$ & $\emptyset$ & $\left\{x_{1}, x_{2}, x_{3}, x_{4}\right\}$ & $\left\{x_{5}, \ldots, x_{16}\right\}$ & \multirow{10}{*}{$\begin{array}{l}\text { Same } \\
\text { results }\end{array}$} \\
\hline & 10 & $\left\{x_{1}, \ldots, x_{9}\right\}$ & $\left\{x_{10}, \ldots, x_{15}\right\}$ & $\emptyset$ & $\left\{x_{1}, \ldots, x_{9}\right\}$ & $\left\{x_{10}, \ldots, x_{15}\right\}$ & \\
\hline \multirow{2}{*}{ DTLZ2 } & 5 & $\left\{x_{1}, x_{2}, x_{3}, x_{4}\right\}$ & $\left\{x_{5}, \ldots, x_{16}\right\}$ & $\emptyset$ & $\left\{x_{1}, x_{2}, x_{3}, x_{4}\right\}$ & $\left\{x_{5}, \ldots, x_{16}\right\}$ & \\
\hline & 10 & $\left\{x_{1}, \ldots, x_{9}\right\}$ & $\left\{x_{10}, \ldots, x_{15}\right\}$ & $\emptyset$ & $\left\{x_{1}, \ldots, x_{9}\right\}$ & $\left\{x_{10}, \ldots, x_{15}\right\}$ & \\
\hline \multirow{2}{*}{ DTLZ3 } & 5 & $\left\{x_{1}, x_{2}, x_{3}, x_{4}\right\}$ & $\left\{x_{5}, \ldots, x_{16}\right\}$ & $\emptyset$ & $\left\{x_{1}, x_{2}, x_{3}, x_{4}\right\}$ & $\left\{x_{5}, \ldots, x_{16}\right\}$ & \\
\hline & 10 & $\left\{x_{1}, \ldots, x_{9}\right\}$ & $\left\{x_{10}, \ldots, x_{15}\right\}$ & $\emptyset$ & $\left\{x_{1}, \ldots, x_{9}\right\}$ & $\left\{x_{10}, \ldots, x_{15}\right\}$ & \\
\hline \multirow{2}{*}{ DTLZ4 } & 5 & $\left\{x_{1}, x_{2}, x_{3}, x_{4}\right\}$ & $\left\{x_{5}, \ldots, x_{16}\right\}$ & $\emptyset$ & $\left\{x_{1}, x_{2}, x_{3}, x_{4}\right\}$ & $\left\{x_{5}, \ldots, x_{16}\right\}$ & \\
\hline & 10 & $\left\{x_{1}, \ldots, x_{9}\right\}$ & $\left\{x_{10}, \ldots, x_{15}\right\}$ & $\emptyset$ & $\left\{x_{1}, \ldots, x_{9}\right\}$ & $\left\{x_{10}, \ldots, x_{15}\right\}$ & \\
\hline \multirow{2}{*}{ DTLZ7 } & 5 & $\left\{x_{1}, x_{2}, x_{3}, x_{4}\right\}$ & $\left\{x_{5}, \ldots, x_{16}\right\}$ & $\emptyset$ & $\left\{x_{1}, x_{2}, x_{3}, x_{4}\right\}$ & $\left\{x_{5}, \ldots, x_{16}\right\}$ & \\
\hline & 10 & $\left\{x_{1}, \ldots, x_{9}\right\}$ & $\left\{x_{10}, \ldots, x_{15}\right\}$ & $\emptyset$ & $\left\{x_{1}, \ldots, x_{9}\right\}$ & $\left\{x_{10}, \ldots, x_{15}\right\}$ & \\
\hline \multirow{2}{*}{ DTLZ5 } & 5 & $\left\{x_{1}, x_{2}, x_{3}, x_{4}\right\}$ & $\left\{x_{7}, x_{9}, x_{10}, x_{11}\right.$, & $\left\{x_{5}, x_{6}, x_{8}, x_{12}, x_{16}\right\}$ & $\left\{x_{1}, x_{2}, x_{3}, x_{4}\right\}$ & $\left\{x_{5}, \ldots, x_{16}\right\}$ & \multirow{6}{*}{$\begin{array}{l}\text { The variables } \\
\text { related to both } \\
\text { convergence } \\
\text { and diversity } \\
\text { are labeled } \\
\text { as convergence } \\
\text { related variables }\end{array}$} \\
\hline & 10 & $\left\{x_{1}, \ldots, x_{9}\right\}$ & $\left\{x_{11}\right\}$ & $\left\{x_{10}, x_{12}, \ldots, x_{15}\right\}$ & $\left\{x_{1}, \ldots, x_{9}\right\}$ & $\left\{x_{10}, \ldots, x_{15}\right\}$ & \\
\hline \multirow{2}{*}{ DTLZ6 } & 5 & $\left\{x_{1}, x_{2}, x_{3}, x_{4}\right\}$ & $\begin{array}{r}\left\{x_{5}, \ldots, x_{11},\right. \\
\left.x_{13}, x_{15}, x_{16}\right\}\end{array}$ & $\left\{x_{12}, x_{14}\right\}$ & $\left\{x_{1}, x_{2}, x_{3}, x_{4}\right\}$ & $\left\{x_{5}, \ldots, x_{16}\right\}$ & \\
\hline & 10 & $\left\{x_{1}, \ldots, x_{9}\right\}$ & $\left\{x_{12}, x_{13}\right\}$ & $\left\{x_{10}, x_{11}, x_{14}, x_{15}\right\}$ & $\left\{x_{1}, \ldots, x_{9}\right\}$ & $\left\{x_{10}, \ldots, x_{15}\right\}$ & \\
\hline \multirow{2}{*}{ WFG3 } & 5 & $\left\{x_{1}, x_{2}, x_{3}, x_{4}\right\}$ & $\left\{x_{8}, \ldots, x_{13}\right.$ & $\left\{x_{5}, x_{6}, x_{7}, x_{14}\right\}$ & $\left\{x_{1}, x_{2}, x_{3}, x_{4}\right\}$ & $\left\{x_{5}, \ldots, x_{16}\right\}$ & \\
\hline & 10 & $\left\{x_{1}, \ldots, x_{9}\right\}$ & $\left\{x_{14}, x_{15}\right\}$ & $\left\{x_{10}, x_{11}, x_{12}, x_{13}\right\}$ & $\left\{x_{1}, \ldots, x_{9}\right\}$ & $\left\{x_{10}, \ldots, x_{15}\right\}$ & \\
\hline UF9 & 3 & $\emptyset$ & $\left\{x_{3}, \ldots, x_{16}\right\}$ & $\left\{x_{1}, x_{2}\right\}$ & $\left\{x_{1}, x_{2}\right\}$ & $\left\{x_{3}, \ldots, x_{16}\right\}$ & \multirow{2}{*}{$\begin{array}{c}\text { Labeled as } \\
\text { diversity related }\end{array}$} \\
\hline UF10 & 3 & $\emptyset$ & $\left\{x_{3}, \ldots, x_{16}\right\}$ & $\left\{x_{1}, x_{2}\right\}$ & $\left\{x_{1}, x_{2}\right\}$ & $\left\{x_{3}, \ldots, x_{16}\right\}$ & \\
\hline
\end{tabular}

It is worth noting that, there are also some other treestructure based non-dominated sorting approaches. For example, the sorting algorithm proposed in [73] stores the non-dominated solutions in a $M$-d tree $(M$ is the number of objectives), and adds new solution via inserting and deleting nodes from the tree; another sorting algorithm proposed in [74] uses a binary tree to store the dominance relationships between solutions, by adjusting the binary tree the solutions in each front can be identified; and the recently proposed M-front [54] keeps an $K$ $\mathrm{d}$ tree to perform approximate nearest neighbor search for determining whether a new solution is dominated by the existing non-dominated solutions. Compared to these existing tree-structure based approaches, the proposed T-ENS holds a considerably less computational complexity as it benefits from the framework of EN$S$ [55], which allows candidate solutions to be inserted into the tree one by one without adjusting the structure of it.

The main idea of T-ENS is to use a tree to represent the solutions in each non-dominated front, where the information about the objectives determining the nondominance relationship between solutions are recorded by the position of nodes in the tree in which the solutions are stored. As a result, many non-dominance relationships between solutions can be inferred from those that have been assigned to the front (stored in the tree), thereby considerably reducing the number of comparisons between solutions belonging to the same front. This leads to considerable enhancement of the computational efficiency of T-ENS over ENS, in particular for solving
MaOPs. Algorithms 6, 7 and 8 present the detailed steps of T-ENS in pseudo code.

To be specific, as done in ENS, T-ENS first sorts the solutions in the population according to an ascending order of the first objective for $M$-objective minimization problems, $M \geq 2$. Let us assume that the sorted population consists of the following $N$ solutions: $p_{1}\left(f_{1}^{1}, f_{2}^{1}, \ldots, f_{M}^{1}\right), p_{2}\left(f_{1}^{2}, f_{2}^{2}, \ldots, f_{M}^{2}\right), \ldots$, $p_{N}\left(f_{1}^{N}, f_{2}^{N}, \ldots, f_{M}^{N}\right)$, where $f_{j}^{i}$ is the $j$-th objective value of the $i$-th solution, $1 \leq j \leq M$ and $1 \leq i \leq N$. Then, T-ENS starts to construct a tree for each non-dominated front. T-ENS adopts the first solution $p_{1}$ as the root of the tree for the first non-dominated front $F_{1}$ and all other solutions in the population belonging to $F_{1}$ will be stored as the descendants of $p_{1}$. The position of solution $p_{i}$, $2 \leq i \leq N$, in the tree is determined by the minimum $j$ $(j>1)$ satisfying that $f_{o b j \operatorname{Seq}[1][j]}^{i}<f_{o b j S e q[1][j]}^{1}$, where objSeq[1][j] is the $j$-th element of a sequence that is randomly generated from 2 to $M$ for solution $p_{1}$. More precisely, a solution $p_{i}$ with the objSeq[1][j]-th objective satisfying the above condition will be stored as the $j$ th child of the root. If there is more than one solution that satisfies the above condition, the next solution will be stored as a child of $p_{i}$, i.e., a grandchild of $p_{1}$. This procedure repeats until all solutions in the population are checked. After the construction of tree for the first non-dominated front $F_{1}$ is complete, T-ENS starts to construct a tree for the second front $F_{2}$ until all solutions in the population are assigned to a tree.

With the above tree constructed to represent solutions in a front, if a solution $p$ to be assigned to the front is 

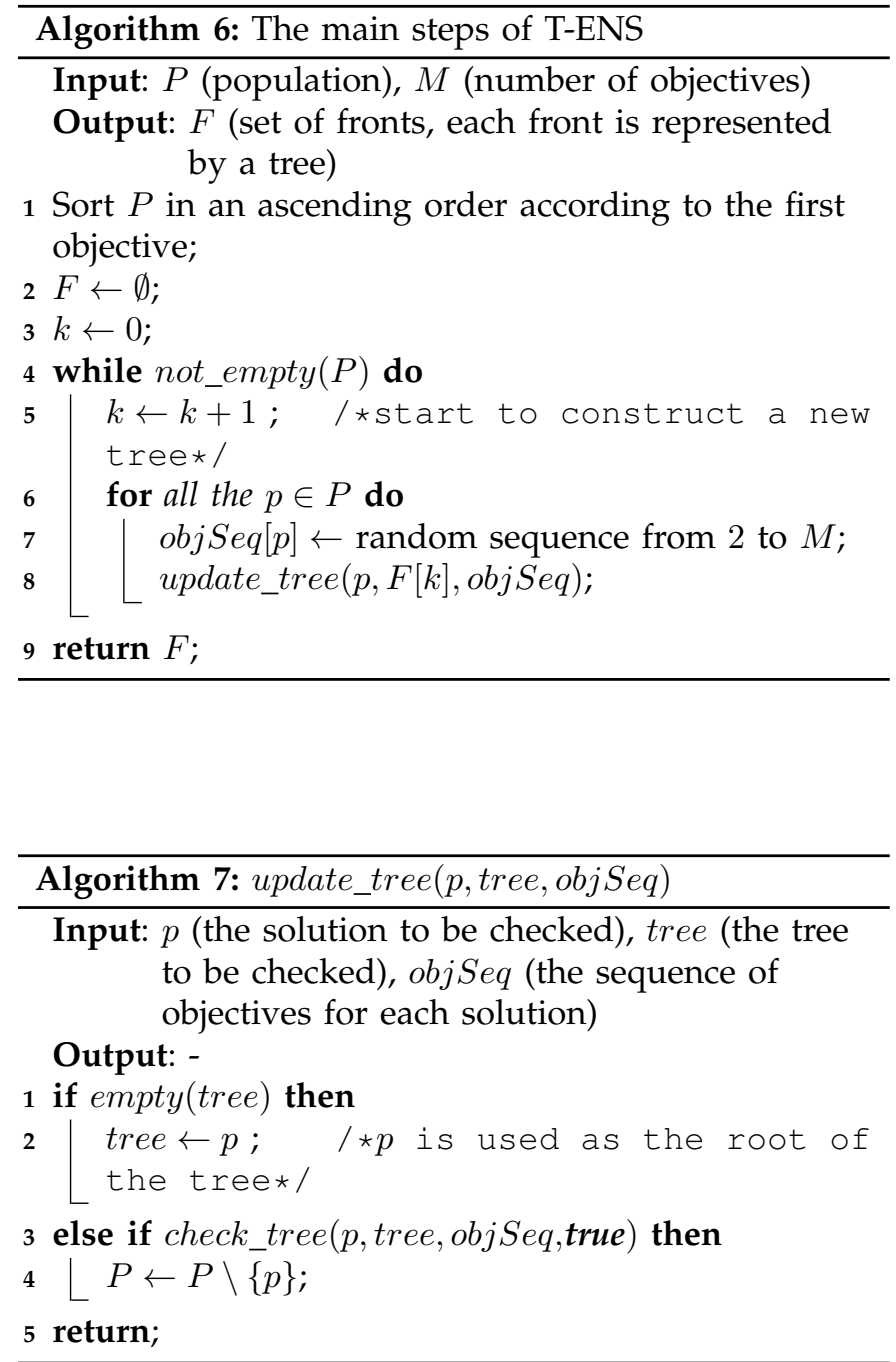

non-dominated with a solution $q$ in the tree, where $p$ has a smaller value than $q$ on the $\operatorname{obj} S e q[q][j]$-th objective ( $p$ has a larger value than $q$ on the first objective since $q$ is ranked before $p$ in the sorted population), then the non-dominance relationship between $p$ and the solution stored as the $k$-th $(k>j)$ child of node $q$ and all descendants of this child can be inferred from the nondominance relationship between $q$ and $p$, since for each of these solutions $s$ we have $f_{o b j S e q[q][j]}^{p}<f_{o b j S e q[q][j]}^{q}<$ $f_{\text {objSeq[q] }[j]}^{s}, j \in\{1,2, \ldots, M-1\}$. Therefore, $p$ does not need to be compared with these solutions in determining the front of $p$, which enables T-ENS work very efficiently for MaOPs.

To investigate the computational efficiency of the proposed T-ENS, we have conducted theoretical time complexity analysis and empirical evaluation which can be found in the supplementary materials. Compared to most existing non-dominated approaches, which hold a time complexity of $O\left(M N^{2}\right)$ on non-dominated populations, T-ENS is computationally more efficient, holding a time complexity of $O(M N \log N / \log M)$, which enables the proposed LMEA to work faster for solving largescale MaOPs.

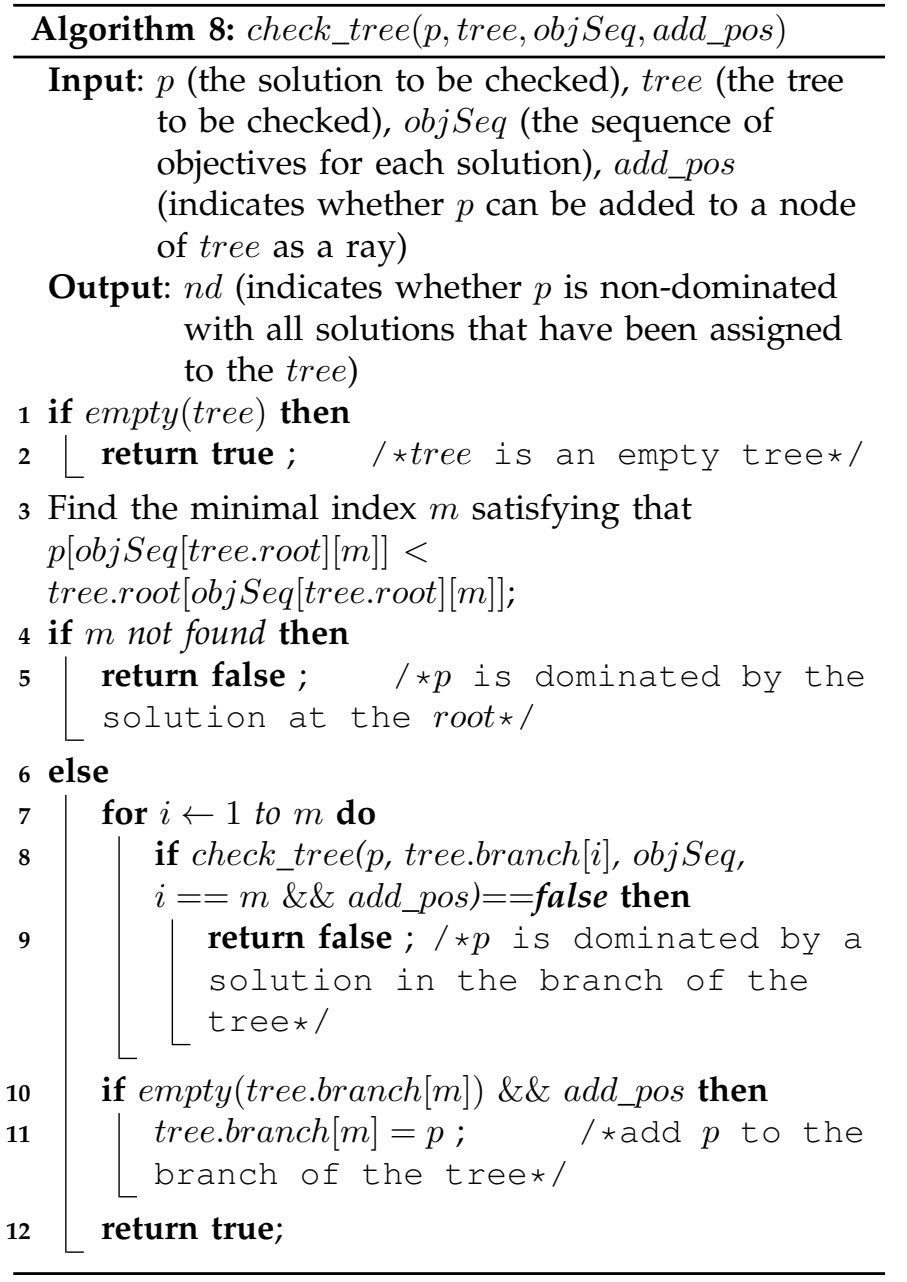

\section{Simulation EXPERIMENTS AND ANALYSIS}

In this section, we verify the performance of LMEA in solving large-scale MaOPs by empirically comparing it with four state-of-the-art MOEAs, namely, MOEA/D [31], NSGA-III [38], KnEA [23] and MOEA/DVA [65]. The MOEA/D, NSGA-III and KnEA are three well-known MOEAs for solving MaOPs, and MOEA/DVA is the first decision variable analysis based MOEA recently proposed in [65] to solve large-scale MOPs.

The experiments are conducted on 10 test problems shown in Table I, which are taken from three widely used test suites, DTLZ [75], WFG [76] and UF [77]. These test problems include DTLZ1 to DTLZ7, WFG3, UF9 and UF10, which can be categorized into three groups according to the decision variable grouping results in Table I. The first group contains DTLZ1 to DTLZ4 and DTLZ7, on which the proposed LMEA and MOEA/DVA have the same grouping results. The second group consists of DTLZ5, DTLZ6 and WFG3, on which the variables related to both convergence and diversity in MOEA/DVA are identified as convergence-related variables in LMEA. The third group contains UF9 and UF10, on which the variables related to both convergence and diversity in MOEA/DVA are identified as diversity-related variables in LMEA. Due to the limitation of pages, we have not 
presented the experimental results on other WFG and UF test problems. Further experiments indicate that all WFG test problems except WFG3 belong to the first group, and all UF test problems belong to the third group. Regarding the number of decision variables, 100, 500 and 1000 decision variables are considered for each test problem, respectively. Regarding the number of objectives, for each DTLZ and WFG test problem, 5 and 10 objectives are considered, respectively, while both UF9 and UF10 are 3-objective. Moreover, the proposed LMEA is further challenged on the test problems with 2000 and 5000 decision variables. In this case, all the test problems used in our simulation experiments are largescale MaOPs, except UF9 and UF10, which are largescale MOPs.

\section{A. Experimental Setting}

For fair comparisons, all compared algorithms adopt the recommended parameter values to achieve the best performance. To be specific, the parameter settings for all conducted experiments are as follows.

1) Crossover and mutation: All compared algorithms adopt the simulated binary crossover (SBX) [68] to create offspring for DTLZ and WFG test problems, and differential evolution (DE) [70], [71], [78] for UF test problems. For each test problem, the polynomial mutation [69] is also performed in all algorithms. The distribution indexes of crossover and mutation are all set to 20 . The crossover probability and mutation probability are set to $p_{c}=1.0$ and $p_{m}=1 / D$, respectively, where $D$ denotes the number of decision variables. The control parameters $F$ and $C R$ in DE are set to $F=0.5$ and $C R=1.0$, as recommended in [78].

2) Population sizing: The population size of NSGA-III and MOEA/D can not be arbitrarily specified, which is equal to the number of uniformly distributed reference points (or weight vectors) generated with the simplex lattice design. As recommended in [38]-[40], the twolayer reference point generation strategy is adopted here. Table II presents the setting of population size in NSGAIII and MOEA/D, where $p_{1}$ and $p_{2}$ are parameters controlling the numbers of reference points along the outer and inner layers of the Pareto front, respectively. To be consistent with NSGA-III and MOEA/D, KnEA, MOEA/DVA and LMEA adopt the same population size settings with respect to the number of objectives.

3) Stopping condition and number of runs: The number of evaluations is used as the termination criterion for all considered algorithms. For each test instance with 100, 500, 1000, 2000 and 5000 decision variables, the maximum number of evaluations is set to $1,000,000,6,800,000$, $17,000,000,50,000,000$ and 230,000,000, respectively. On each test instance, 20 independent runs are performed for each algorithm to obtain statistical results.

4) Other parameters: The range of neighborhood, the maximal number of solutions replaced by each child solution and the probability that parent solutions are
TABLE II

SetTing of Population Size In NSGA-III ANd MOEA/D, Where $p_{1}$ AND $p_{2}$ Are Parameters Controlling the Numbers of REFERENCE POINTS ALONG THE BOUNDARY OF THE PARETO Front AND INSIDE IT, RESPECTIVELY.

\begin{tabular}{ccc}
\hline $\begin{array}{c}\text { Number of } \\
\text { objectives }\end{array}$ & Parameter $\left(p_{1}, p_{2}\right)$ & Population size \\
\hline 3 & $(13,0)$ & 105 \\
5 & $(5,0)$ & 126 \\
10 & $(3,2)$ & 275 \\
\hline
\end{tabular}

selected from the neighborhood in MOEA/D (as well as MOEA/DVA) are set to $T=0.1 N, n_{r}=0.01 N$ and $\delta=0.9$ for all test problems, respectively, where $N$ is the population size. The Tchebycheff approach is employed as the aggregation function in MOEA/D. For KnEA, the parameter $T$ for controlling the ratio of knee points is set to 0.3 for 5-objective DTLZ1 and DTLZ3, 0.4 for 5objective DTLZ5 and DTLZ6, 0.5 for 5-objective DTLZ2, DTLZ4 and DTLZ7, as well as UF9 and UF10; $T$ is set to the recommended values for 10-objective DTLZ and WFG test problems [23]. For MOEA/DVA, the number of interaction analysis and the number of control property analysis are set to the recommended values, namely, $N I A=6$ and $N C A=50$. For LMEA, the number of selected solutions and the number of perturbations for each selected solution in decision variable clustering are set to $n S e l=2$ and $n P e r=4$, respectively, and the number of selected solutions in decision variable interaction analysis is set to $n C o r=6$. A detailed sensitivity analysis of LMEA to parameters $n \mathrm{Sel}$ and $n P e r$ can be found in the supplementary materials.

4) Performance metric: Two widely used performance indicators, the inverted generational distance (IGD) [79] and the hypervolume (HV) [80], [81] are adopted to evaluate the performance of the compared algorithms. As recommended in [38], [40], the reference point sets required for IGD calculations are generated by sampling uniformly distributed points using the Das and Dennis method [82], and a set size closest to 5000 is used in this work for all test instances. For the calculation of HV, we first discard the solutions that are outside the box bounded by the ideal point and nadir point of the true Pareto front, and then normalize the remaining solutions inside. Since the same reference point $(1,1, \ldots, 1)$ is used for all test instances, it is guaranteed that all the obtained HV values will be inside the interval $[0,1]$. Besides, we adopt the exact calculation for test problems with 3 objectives and the Monte Carlo estimation method when the number of objective is larger than 3 . Note that both IGD and HV can account for the convergence as well as the distribution of a solution set. A smaller value of IGD indicates a better quality of the obtained solution set, while a larger value of HV signals a better quality. 
TABLE III

IGD Metric Values of the Five Algorithms on DTlZ1, DTLZ2, DTLZ3, DTLZ4 and DTLZ7, Where the Best Result on Each Test INSTANCE IS SHOWN IN A GRAY BACKGROUND.

\begin{tabular}{|c|c|c|c|c|c|c|c|}
\hline Problem & Obj. & Dec. & MOEA/D & NSGA-III & KnEA & MOEA/DVA & LMEA \\
\hline \multirow{6}{*}{ DTLZ1 } & \multirow{3}{*}{5} & 100 & $1.1859 \mathrm{e}-1(1.63 \mathrm{e}-3)-$ & $3.9340 \mathrm{e}+0(1.72 \mathrm{e}+0)-$ & $6.6202 \mathrm{e}+0(1.83 \mathrm{e}+0)-$ & $6.2932 \mathrm{e}-2(5.72 \mathrm{e}-5)-$ & $5.9982 \mathrm{e}-2(4.22 \mathrm{e}-4)$ \\
\hline & & 500 & $1.4648 \mathrm{e}-1(4.30 \mathrm{e}-2)-$ & $5.2597 e+1(5.63 e+0)-$ & $7.6436 e+1(6.86 e+0)-$ & $6.3284 \mathrm{e}-2(1.63 \mathrm{e}-4)-$ & $6.1124 \mathrm{e}-2(5.16 \mathrm{e}-4)$ \\
\hline & & 1000 & $1.6833 \mathrm{e}-1(4.29 \mathrm{e}-2)-$ & $1.1669 \mathrm{e}+2(5.08 \mathrm{e}+0)-$ & $1.3392 \mathrm{e}+2(1.28 \mathrm{e}+1)-$ & $6.3442 \mathrm{e}-2(1.26 \mathrm{e}-4)-$ & $6.0423 e-2(4.09 e-4)$ \\
\hline & \multirow{3}{*}{10} & 100 & $2.2187 \mathrm{e}+0(4.72 \mathrm{e}+0)-$ & $1.1944 \mathrm{e}+2(2.72 \mathrm{e}+1)-$ & $5.5749 \mathrm{e}+0(1.53 \mathrm{e}+0)-$ & $1.4356 \mathrm{e}-1(1.79 \mathrm{e}-2) \approx$ & $1.6302 \mathrm{e}-1(4.87 \mathrm{e}-3)$ \\
\hline & & 500 & $1.6987 \mathrm{e}+2(1.01 \mathrm{e}+2)-$ & $3.9829 \mathrm{e}+2(2.77 \mathrm{e}+1)-$ & $5.0951 \mathrm{e}+1(1.25 \mathrm{e}+1)-$ & $1.7047 \mathrm{e}-1(1.14 \mathrm{e}-2) \approx$ & $1.5995 \mathrm{e}-1(4.05 \mathrm{e}-3)$ \\
\hline & & 1000 & $4.8922 \mathrm{e}+2(2.04 \mathrm{e}+2)-$ & $7.9841 \mathrm{e}+2(4.16 \mathrm{e}+1)-$ & $1.4841 \mathrm{e}+2(1.93 \mathrm{e}+1)-$ & $1.3805 \mathrm{e}-1(2.18 \mathrm{e}-2) \approx$ & $1.6002 \mathrm{e}-1(5.24 \mathrm{e}-3)$ \\
\hline \multirow{6}{*}{ DTLZ2 } & \multirow{3}{*}{5} & 100 & $3.2006 \mathrm{e}-1(2.26 \mathrm{e}-8)-$ & $1.9494 \mathrm{e}-1(7.98 \mathrm{e}-7)-$ & $2.2045 \mathrm{e}-1(1.18 \mathrm{e}-2)-$ & $1.9493 \mathrm{e}-1(9.26 \mathrm{e}-8)-$ & $1.8825 \mathrm{e}-1(2.14 \mathrm{e}-3)$ \\
\hline & & 500 & $3.2006 \mathrm{e}-1(5.31 \mathrm{e}-8)-$ & $1.9494 \mathrm{e}-1(5.62 \mathrm{e}-8)-$ & $2.3629 e-1(7.63 e-3)-$ & $1.9494 \mathrm{e}-1(3.17 \mathrm{e}-8)-$ & $1.8832 \mathrm{e}-1(2.29 \mathrm{e}-3)$ \\
\hline & & 1000 & $3.2006 \mathrm{e}-1(1.12 \mathrm{e}-7)-$ & $1.9494 \mathrm{e}-1(9.86 \mathrm{e}-7)-$ & $2.2844 \mathrm{e}-1(1.00 \mathrm{e}-2)-$ & $1.9494 \mathrm{e}-1(6.25 \mathrm{e}-8)-$ & $1.8816 e-1(2.53 e-3)$ \\
\hline & \multirow{3}{*}{10} & 100 & $7.1528 \mathrm{e}-1(1.81 \mathrm{e}-2)-$ & $4.2141 \mathrm{e}-1(1.58 \mathrm{e}-4)+$ & $4.1230 \mathrm{e}-1(2.24 \mathrm{e}-3)+$ & $4.5923 \mathrm{e}-1(5.13 \mathrm{e}-2) \approx$ & $5.0905 \mathrm{e}-1(1.48 \mathrm{e}-2)$ \\
\hline & & 500 & $7.2369 e-1(8.63 e-3)-$ & $4.2152 e-1(4.79 e-5)+$ & $4.1402 \mathrm{e}-1(5.07 \mathrm{e}-3)+$ & $4.1976 \mathrm{e}-1(3.53 \mathrm{e}-4)+$ & $5.0617 \mathrm{e}-1(1.78 \mathrm{e}-2)$ \\
\hline & & 1000 & 7.3217e-1(1.90e-2)- & $4.2172 \mathrm{e}-1(5.76 \mathrm{e}-5)+$ & $4.1222 \mathrm{e}-1(1.85 \mathrm{e}-3)+$ & $4.2065 \mathrm{e}-1(1.19 \mathrm{e}-4)+$ & $5.0689 \mathrm{e}-1(2.84 \mathrm{e}-2)$ \\
\hline \multirow{6}{*}{ DTLZ3 } & \multirow{3}{*}{5} & 100 & $3.2877 \mathrm{e}-1(2.49 \mathrm{e}-3)-$ & $2.2868 \mathrm{e}+1(6.14 \mathrm{e}+0)-$ & $7.6962 \mathrm{e}-1(4.18 \mathrm{e}-1)-$ & $1.9505 e-1(5.92 e-5)-$ & $1.8985 \mathrm{e}-1(2.14 \mathrm{e}-3)$ \\
\hline & & 500 & $4.1776 \mathrm{e}-1(1.97 \mathrm{e}-1)-$ & $2.9421 \mathrm{e}+2(3.34 \mathrm{e}+1)-$ & $6.1228 \mathrm{e}-1(1.26 \mathrm{e}-1)-$ & $1.9536 \mathrm{e}-1(1.01 \mathrm{e}-4) \approx$ & $1.9035 \mathrm{e}-1(4.44 \mathrm{e}-3)$ \\
\hline & & 1000 & $4.4681 \mathrm{e}-1(1.40 \mathrm{e}-2)-$ & $6.8229 \mathrm{e}+2(5.50 \mathrm{e}+1)-$ & $8.2477 \mathrm{e}-1(2.42 \mathrm{e}-1)-$ & $1.9563 \mathrm{e}-1(3.70 \mathrm{e}-4) \approx$ & $1.8812 \mathrm{e}-1(4.10 \mathrm{e}-3)$ \\
\hline & \multirow{3}{*}{10} & 100 & $8.0019 \mathrm{e}-1(4.87 \mathrm{e}-2)-$ & $8.2193 e+2(1.37 e+2)-$ & $6.3652 \mathrm{e}+0(3.88 \mathrm{e}+0)-$ & $5.0747 \mathrm{e}-1(3.77 \mathrm{e}-2) \approx$ & $5.5352 \mathrm{e}-1(3.56 \mathrm{e}-2)$ \\
\hline & & 500 & $1.2941 \mathrm{e}+2(2.78 \mathrm{e}+2)-$ & $2.2891 \mathrm{e}+3(1.35 \mathrm{e}+2)-$ & $9.4498 \mathrm{e}+0(4.57 \mathrm{e}+0)-$ & $5.2820 \mathrm{e}-1(9.88 \mathrm{e}-2) \approx$ & $5.5126 \mathrm{e}-1(1.66 \mathrm{e}-2)$ \\
\hline & & 1000 & $2.5643 \mathrm{e}+2(3.27 \mathrm{e}+2)-$ & $4.4071 \mathrm{e}+3(1.91 \mathrm{e}+2)-$ & $4.4621 \mathrm{e}+0(2.34 \mathrm{e}+0)-$ & $4.7728 \mathrm{e}-1(4.26 \mathrm{e}-2)+$ & $5.4964 \mathrm{e}-1(1.85 \mathrm{e}-2)$ \\
\hline \multirow{6}{*}{ DTLZ4 } & \multirow{3}{*}{5} & 100 & $6.2588 \mathrm{e}-1(2.50 \mathrm{e}-1)-$ & $2.7298 \mathrm{e}-1(1.31 \mathrm{e}-1)+$ & $2.1434 \mathrm{e}-1(4.33 \mathrm{e}-3)+$ & $2.6957 \mathrm{e}-1(1.29 \mathrm{e}-1) \approx$ & $2.6411 \mathrm{e}-1(1.55 \mathrm{e}-2)$ \\
\hline & & 500 & $5.2727 \mathrm{e}-1(1.18 \mathrm{e}-1)-$ & $1.9496 \mathrm{e}-1(2.64 \mathrm{e}-5)+$ & $2.1571 \mathrm{e}-1(9.97 \mathrm{e}-3)+$ & $3.4421 \mathrm{e}-1(1.29 \mathrm{e}-1) \approx$ & $2.7256 \mathrm{e}-1(2.46 \mathrm{e}-2)$ \\
\hline & & 1000 & $4.3848 \mathrm{e}-1(1.80 \mathrm{e}-1)-$ & $2.2829 \mathrm{e}-1(1.09 \mathrm{e}-1) \approx$ & $2.1380 \mathrm{e}-1(4.21 \mathrm{e}-3)+$ & $3.4420 \mathrm{e}-1(1.29 \mathrm{e}-1) \approx$ & $2.7071 \mathrm{e}-1(2.36 \mathrm{e}-2)$ \\
\hline & \multirow{3}{*}{10} & 100 & $8.3550 \mathrm{e}-1(3.18 \mathrm{e}-2)-$ & $4.2123 \mathrm{e}-1(1.95 \mathrm{e}-4)+$ & $4.2764 \mathrm{e}-1(2.53 \mathrm{e}-2)+$ & $4.3772 \mathrm{e}-1(3.33 \mathrm{e}-2)+$ & $5.0820 \mathrm{e}-1(2.47 \mathrm{e}-2)$ \\
\hline & & 500 & $8.3052 \mathrm{e}-1(2.93 \mathrm{e}-2)-$ & $4.2154 \mathrm{e}-1(1.02 \mathrm{e}-4)+$ & $4.0104 \mathrm{e}-1(4.57 \mathrm{e}-3)+$ & $4.1970 \mathrm{e}-1(5.83 \mathrm{e}-5)+$ & $5.2786 \mathrm{e}-1(3.98 \mathrm{e}-3)$ \\
\hline & & 1000 & $8.2082 \mathrm{e}-1(2.40 \mathrm{e}-2)-$ & $4.2173 e-1(7.92 e-5)+$ & $4.0081 \mathrm{e}-1(2.61 \mathrm{e}-3)+$ & $4.5695 \mathrm{e}-1(3.18 \mathrm{e}-2)+$ & $5.2345 \mathrm{e}-1(9.26 \mathrm{e}-3)$ \\
\hline \multirow{6}{*}{ DTLZ7 } & \multirow{3}{*}{5} & 100 & $5.2987 \mathrm{e}-1(2.57 \mathrm{e}-2)-$ & $5.2849 \mathrm{e}-1(1.56 \mathrm{e}-1)-$ & $2.4790 \mathrm{e}-1(1.14 \mathrm{e}-2)+$ & $5.2044 \mathrm{e}-1(2.51 \mathrm{e}-6)-$ & $3.0913 \mathrm{e}-1(1.10 \mathrm{e}-2)$ \\
\hline & & 500 & 5.1542e-1(3.73e-7)- & $2.3928 \mathrm{e}+0(1.60 \mathrm{e}-1)-$ & $2.3191 \mathrm{e}-1(7.58 \mathrm{e}-3)+$ & $5.2043 \mathrm{e}-1(4.57 \mathrm{e}-7)-$ & $3.2032 \mathrm{e}-1(8.53 \mathrm{e}-3)$ \\
\hline & & 1000 & $5.2120 \mathrm{e}-1(1.78 \mathrm{e}-2)-$ & $2.6633 e+0(1.38 \mathrm{e}-1)-$ & $2.3408 \mathrm{e}-1(1.44 \mathrm{e}-2)+$ & $5.2043 \mathrm{e}-1(7.54 \mathrm{e}-7)-$ & $3.1051 \mathrm{e}-1(7.59 \mathrm{e}-3)$ \\
\hline & \multirow{3}{*}{10} & 100 & $4.4020 \mathrm{e}+0(1.37 \mathrm{e}+0)-$ & $4.6684 \mathrm{e}+0(4.21 \mathrm{e}-1)-$ & $1.3854 \mathrm{e}+0(3.48 \mathrm{e}-2)-$ & $1.6385 \mathrm{e}+0(7.84 \mathrm{e}-2)-$ & $1.0749 \mathrm{e}+0(6.40 \mathrm{e}-3)$ \\
\hline & & 500 & $5.3581 e+0(6.94 e-1)-$ & $1.1875 e+1(8.36 e-1)-$ & $1.3572 \mathrm{e}+0(1.65 \mathrm{e}-2)-$ & $1.6320 \mathrm{e}+0(1.02 \mathrm{e}-1)-$ & $1.0752 \mathrm{e}+0(3.29 \mathrm{e}-3)$ \\
\hline & & 1000 & $5.7150 \mathrm{e}+0(2.69 \mathrm{e}-1)-$ & $1.5027 e+1(9.39 e-1)-$ & $1.3300 \mathrm{e}+0(9.50 \mathrm{e}-3)-$ & $1.5249 \mathrm{e}+0(4.16 \mathrm{e}-2)-$ & $1.0781 e+0(4.58 \mathrm{e}-3)$ \\
\hline +1 & $/ \approx$ & & $0 / 30 / 0$ & $8 / 21 / 1$ & $12 / 18 / 0$ & $6 / 13 / 11$ & \\
\hline
\end{tabular}

' $+{ }^{\prime},{ }^{\prime}-{ }^{\prime}$ and ${ }^{\prime} \approx$ ' indicate that the result is significantly better, significantly worse and statistically similar to that of LMEA, respectively.

B. Performance Comparison Between LMEA and Existing MOEAs on Large-Scale MaOPs

Tables III and IV present the statistical results of the IGD metric values of the five compared algorithms on the 10 test problems with 100, 500 and 1000 decision variables obtained via 20 independent runs, where the best result on each test instance is shown in a gray background. The Wilcoxon rank sum test is also adopted at a significance level of 0.05 , where the symbols ' + , ' - ' and ' $\approx$ ' indicate that the result is significantly better, significantly worse and statistically similar to that obtained by LMEA, respectively. From the tables, the following three observations can be made. First, the MOEA/D, NSGA-III and KnEA can achieve a promising performance on some of the large-scale test instances under consideration, but their general performance will be worse than that of MOEA/DVA and the proposed LMEA that are specifically designed for solving largescale problems. As can be seen from the tables, the IGD values obtained by MOEA/DVA and LMEA on each test problem are consistently good as the number of decision variables increases from 100 to 1000 , which confirms a promising scalability of MOEA/DVA and LMEA.

Second, on the group of test problems DTLZ1 to DTLZ4 and DTLZ7 where MOEA/DVA and LMEA obtain the same decision variable grouping results, the proposed LMEA has achieved a similar performance as that of MOEA/DVA with the only exception of DTLZ7. 
TABLE IV

IGD Metric Values of the Five Algorithms on DTLZ5, DTLZ6, WFG3, UF9 ANd UF10, Where the Best Result on EACH Test INSTANCE IS SHOWN IN A GRAY BACKGROUND.

\begin{tabular}{|c|c|c|c|c|c|c|c|}
\hline Problem & Obj. & Dec. & MOEA/D & NSGA-III & KnEA & MOEA/DVA & LMEA \\
\hline \multirow{3}{*}{ DTLZ5 } & 5 & 500 & $4.5161 \mathrm{e}-2(1.04 \mathrm{e}-6)-$ & $1.9413 \mathrm{e}-1(1.78 \mathrm{e}-2)-$ & $3.1740 \mathrm{e}-1(6.11 \mathrm{e}-2)-$ & $2.0469 \mathrm{e}-1(5.20 \mathrm{e}-8)-$ & $4.0861 \mathrm{e}-3(1.48 \mathrm{e}-4)$ \\
\hline & \multirow[b]{2}{*}{10} & 100 & $4.9994 \mathrm{e}-2(2.41 \mathrm{e}-4)-$ & $3.1946 \mathrm{e}-1(2.03 \mathrm{e}-2)-$ & $3.6432 \mathrm{e}-1(5.71 \mathrm{e}-2)-$ & $1.8877 \mathrm{e}-1(1.87 \mathrm{e}-4)-$ & $2.3954 e-3(6.95 e-5)$ \\
\hline & & 500 & $5.0407 \mathrm{e}-2(4.16 \mathrm{e}-4)-$ & $5.2642 \mathrm{e}-1(2.04 \mathrm{e}-2)-$ & $3.6389 \mathrm{e}-1(5.43 \mathrm{e}-2)-$ & $1.8866 \mathrm{e}-1(3.30 \mathrm{e}-4)-$ & $2.2721 e-3(4.47 e-5)$ \\
\hline \multirow{5}{*}{ DTLZ6 } & \multirow{3}{*}{5} & 100 & $1.4970 \mathrm{e}-1(3.14 \mathrm{e}-2)-$ & $2.5642 \mathrm{e}-1(2.29 \mathrm{e}-2)-$ & $5.8811 \mathrm{e}-1(1.34 \mathrm{e}-1)-$ & $1.8236 \mathrm{e}-1(2.43 \mathrm{e}-6)-$ & $3.9943 e-3(2.14 e-4)$ \\
\hline & & 500 & $1.3010 \mathrm{e}+0(1.04 \mathrm{e}-1)-$ & $4.9939 \mathrm{e}-1(1.89 \mathrm{e}-2)-$ & $7.2754 \mathrm{e}-1(1.43 \mathrm{e}-1)-$ & $1.8236 \mathrm{e}-1(4.25 \mathrm{e}-7)-$ & $4.5127 \mathrm{e}-3(1.22 \mathrm{e}-3)$ \\
\hline & & 1000 & $2.7140 \mathrm{e}+0(1.97 \mathrm{e}-1)-$ & $6.5774 \mathrm{e}-1(2.19 \mathrm{e}-2)-$ & $1.5085 \mathrm{e}+0(4.53 \mathrm{e}-1)-$ & $1.8236 \mathrm{e}-1(5.91 \mathrm{e}-7)-$ & $3.9747 \mathrm{e}-3(2.29 \mathrm{e}-4)$ \\
\hline & \multirow[b]{2}{*}{10} & 100 & $6.7510 \mathrm{e}-2(1.85 \mathrm{e}-2)-$ & $7.2120 \mathrm{e}+0(1.35 \mathrm{e}+0)-$ & $3.7560 \mathrm{e}+0(9.56 \mathrm{e}-1)-$ & $1.6531 \mathrm{e}-1(4.09 \mathrm{e}-2)-$ & $2.4477 \mathrm{e}-3(5.11 \mathrm{e}-4)$ \\
\hline & & 500 & $1.1735 \mathrm{e}+0(2.52 \mathrm{e}-1)-$ & $8.7171 \mathrm{e}+1(4.88 \mathrm{e}+0)-$ & $6.3085 \mathrm{e}+0(2.18 \mathrm{e}+0)-$ & $1.2750 \mathrm{e}-1(5.33 \mathrm{e}-2)-$ & $3.0711 \mathrm{e}-3(7.20 \mathrm{e}-4)$ \\
\hline \multirow{4}{*}{ WFG3 } & 5 & 1000 & $2.3370 \mathrm{e}+0(8.10 \mathrm{e}-2)-$ & $8.8753 \mathrm{e}-1(2.53 \mathrm{e}-2)-$ & $4.6414 \mathrm{e}-1(1.49 \mathrm{e}-1)-$ & $2.4410 \mathrm{e}+0(3.52 \mathrm{e}-2)-$ & $1.2493 \mathrm{e}-1(2.41 \mathrm{e}-2)$ \\
\hline & \multirow{3}{*}{10} & 100 & $3.4569 \mathrm{e}+0(1.29 \mathrm{e}-1)-$ & $3.0344 \mathrm{e}+0(5.71 \mathrm{e}-2)-$ & $2.2907 \mathrm{e}+0(7.93 \mathrm{e}-1)-$ & $3.4846 \mathrm{e}+0(2.45 \mathrm{e}-2)-$ & $1.8542 \mathrm{e}-1(5.96 \mathrm{e}-2)$ \\
\hline & & 500 & $3.8106 \mathrm{e}+0(8.24 \mathrm{e}-2)-$ & $3.1112 \mathrm{e}+0(5.04 \mathrm{e}-2)-$ & $1.6148 e+0(5.53 e-1)-$ & $3.5264 \mathrm{e}+0(9.75 \mathrm{e}-2)-$ & $4.8685 \mathrm{e}-1(5.49 \mathrm{e}-2)$ \\
\hline & & 1000 & $3.9456 \mathrm{e}+0(7.23 \mathrm{e}-2)-$ & $3.1454 \mathrm{e}+0(4.20 \mathrm{e}-2)-$ & $1.9861 \mathrm{e}+0(1.27 \mathrm{e}+0)-$ & $3.5070 \mathrm{e}+0(1.17 \mathrm{e}-1)-$ & $6.9330 \mathrm{e}-1(1.16 \mathrm{e}-1)$ \\
\hline \multirow{3}{*}{ UF9 } & \multirow{3}{*}{3} & 100 & $2.9851 \mathrm{e}-1(1.58 \mathrm{e}-2)-$ & $2.2030 \mathrm{e}-1(9.19 \mathrm{e}-2)-$ & $5.3546 \mathrm{e}-1(1.39 \mathrm{e}-1)-$ & $4.3517 \mathrm{e}-2(2.50 \mathrm{e}-6)+$ & $5.7008 \mathrm{e}-2(8.91 \mathrm{e}-3)$ \\
\hline & & 500 & $3.1975 \mathrm{e}-1(2.92 \mathrm{e}-2)-$ & $3.1029 \mathrm{e}-1(7.27 \mathrm{e}-2)-$ & 4.6017e-1(1.19e-1)- & $4.3516 \mathrm{e}-2(9.76 \mathrm{e}-7)+$ & $5.3626 \mathrm{e}-2(6.94 \mathrm{e}-3)$ \\
\hline & & 1000 & $3.0557 \mathrm{e}-1(8.39 \mathrm{e}-2)-$ & $3.7850 \mathrm{e}-1(4.21 \mathrm{e}-2)-$ & $5.3607 \mathrm{e}-1(8.03 \mathrm{e}-2)-$ & $4.3516 \mathrm{e}-2(7.00 \mathrm{e}-7)+$ & $5.1231 \mathrm{e}-2(4.50 \mathrm{e}-3)$ \\
\hline \multirow[b]{2}{*}{ UF10 } & \multirow[b]{2}{*}{3} & 100 & $5.9354 \mathrm{e}-1(1.50 \mathrm{e}-1)-$ & $3.3482 \mathrm{e}-1(8.13 \mathrm{e}-2)-$ & $7.5510 \mathrm{e}-1(1.49 \mathrm{e}-1)-$ & $1.1024 \mathrm{e}-1(2.92 \mathrm{e}-3)+$ & $1.6632 \mathrm{e}-1(1.45 \mathrm{e}-2)$ \\
\hline & & 500 & $6.3119 \mathrm{e}-1(1.92 \mathrm{e}-1)-$ & $3.6779 \mathrm{e}-1(8.36 \mathrm{e}-2)-$ & $1.3142 \mathrm{e}+0(8.69 \mathrm{e}-1)-$ & $1.0158 \mathrm{e}-1(8.55 \mathrm{e}-4)+$ & $1.5547 \mathrm{e}-1(4.99 \mathrm{e}-3)$ \\
\hline
\end{tabular}

' $+{ }^{\prime},{ }^{\prime}-{ }^{\prime}$ and ${ }^{\prime} \approx$ ' indicate that the result is significantly better, significantly worse and statistically similar to that of LMEA, respectively.

On all the DTLZ7 test instances, the proposed LMEA always obtains a smaller IGD value than MOEA/DVA. This is attributed to the fact that MOEA/DVA adopts MOEA/D for optimizing the diversity-related variables, while such decomposition based MOEAs are not well suited for solving MOPs or MaOPs with irregular (disconnected or degenerate) Pareto optimal fronts [38], [40], [83]. By contrast, the distribution management strategy as detailed in Algorithm 4 is capable of handling such irregular Pareto optimal fronts as that of DTLZ7.

Finally, on the group of test problems DTLZ5, DTLZ6, WFG3, UF9 and UF10 where MOEA/DVA and LMEA obtain different decision variable grouping results, LMEA shows generally better performance than MOEA/DVA in terms of IGD. As can be seen from Table IV, on DTLZ5, DTLZ6 and WFG3, the variables related to both convergence and diversity in MOEA/DVA are identified as convergence-related variables in LMEA, thus resulting in significantly smaller IGD values. This observation confirms that the proposed variable clustering strategy in LMEA is able to reasonably identify the decision variables related to both convergence and diversity, which can not be distinguished by MOEA/DVA.

It is worth noting that, MOEA/DVA and LMEA still achieve similar performance on UF9 and UF10, although different decision variable grouping results have been obtained by both algorithms. This is due to the fact that MOEA/DVA optimizes all variables related to both convergence and diversity, e.g., $x_{1}$ and $x_{2}$ in UF9 and UF10, in the same way as diversity-related variables. As shown in Table I, the variables $x_{1}$ and $x_{2}$ in UF9 and UF10 are identified as diversity-related variables in LMEA. The slightly better performance of MOEA/DVA than LMEA on UF9 and UF10 is due to the fact that MOEA/DVA adopts a uniform sampling method developed in [84] to initialize the population of diversityrelated variables, which is well suited for maintaining the distribution of the candidate solutions when applied to the UF problems.

For further observations, Fig. 4 plots the nondominated solutions with the best IGD metric values obtained by the five algorithms among 20 runs in 
TABLE V

Runtime (s) Of the Five Algorithms Averaging Over All Test Instances with 100, 500 AND 1000 Variables.

\begin{tabular}{|c|c|c|c|c|c|c|c|c|c|}
\hline \multirow[b]{2}{*}{$\begin{array}{c}\text { Number of } \\
\text { variables }\end{array}$} & \multirow[b]{2}{*}{ MOEA/D } & \multirow[b]{2}{*}{ NSGA-III } & \multirow[b]{2}{*}{ KnEA } & \multicolumn{3}{|c|}{ MOEA/DVA } & \multicolumn{3}{|c|}{ LMEA } \\
\hline & & & & $\begin{array}{l}\text { Control } \\
\text { property } \\
\text { analysis }\end{array}$ & $\begin{array}{c}\text { Interaction } \\
\text { analysis }\end{array}$ & Search & $\begin{array}{l}\text { Variable } \\
\text { clustering }\end{array}$ & $\begin{array}{c}\text { Interaction } \\
\text { analysis }\end{array}$ & Search \\
\hline 100 & $1.0499 \mathrm{e}+2$ & $9.2768 \mathrm{e}+1$ & $2.2953 e+2$ & $2.0693 \mathrm{e}-1$ & $2.0669 \mathrm{e}+1$ & $1.4598 \mathrm{e}+2$ & $1.4638 \mathrm{e}-1$ & $1.4218 \mathrm{e}+1$ & $3.7283 e+1$ \\
\hline 500 & $7.4233 \mathrm{e}+2$ & $2.5760 \mathrm{e}+3$ & $1.6144 \mathrm{e}+3$ & $1.2292 \mathrm{e}+0$ & $5.3845 \mathrm{e}+2$ & $7.6295 \mathrm{e}+2$ & $7.9235 \mathrm{e}-1$ & $4.0784 \mathrm{e}+2$ & $1.7208 \mathrm{e}+2$ \\
\hline 1000 & $1.8008 \mathrm{e}+3$ & $7.4880 \mathrm{e}+3$ & $4.2560 \mathrm{e}+3$ & $2.9508 \mathrm{e}+0$ & $2.3653 e+3$ & $1.2397 \mathrm{e}+3$ & $1.4735 \mathrm{e}+0$ & $1.9820 \mathrm{e}+3$ & $4.0282 \mathrm{e}+2$ \\
\hline
\end{tabular}



(a)

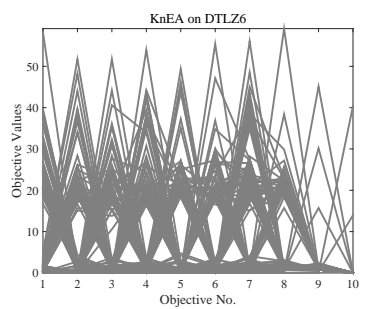

(c)

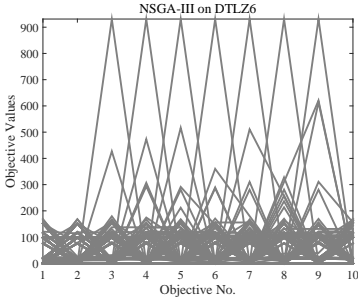

(b)

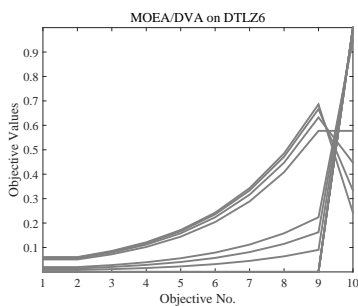

(d)

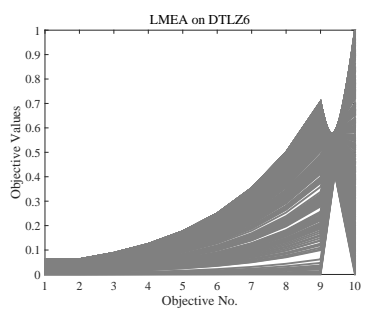

(e)

Fig. 4. The non-dominated solutions with the lowest IGD metric values obtained by each algorithm among 20 runs in the objective space on 10-objective DTLZ6 problem with 1000 decision variables.

the objective space on 10-objective DTLZ6 with 1000 decision variables, respectively. It can clearly be seen that the proposed LMEA and MOEA/DVA outperform MOEA/D, NSGA-III and KnEA on 10-objective DTLZ6 with 1000 decision variables in terms of both convergence and diversity, and the LMEA achieves much more non-dominated solutions in the Pareto optimal front than MOEA/DVA, which confirms the effectiveness of decision variable clustering method developed in LMEA.

To verify the computational efficiency of LMEA, Table V summarizes the runtime(s) of the five compared algorithms averaged over all the runs on test instances with 100, 500 and 1000 variables, respectively. Note that the proposed T-ENS is employed as the non-dominated approach in all compared algorithms except MOEA/D.
The runtime of LMEA and MOEA/DVA consists of three parts, namely, runtime of decision variable clustering (known as control property analysis in MOEA/DVA), runtime of decision variable interaction analysis and runtime of search stage. It can be observed from the table that LMEA is computationally more efficient than MOEA/D, NSGA-III, KnEA and MOEA/DVA on test problems with 100 and 500 variables, while MOEA/D performs the best on problems with 1000 variables. For LMEA, the variable clustering component is less timeconsuming than the other two components, namely decision variable interaction analysis and the search stage. In addition, on the one hand, the runtime of search stage in LMEA is almost linear scaling to the number of decision variables; on the other hand, however, the runtime of variable interaction analysis rapidly increases with the number of decision variables, which is due to the fact that this component holds a time complexity of $O\left(D^{2}\right)$ in the worst case, where $D$ denotes the number of variables.

Based on the above empirical results, we can conclude that the proposed LMEA is more effective and efficient to handle large-scale MaOPs than state-of-the-art MOEAs designed for solving MaOPs or large-scale MOPs.

\section{Performance of LMEA on Large-scale MaOPs with 2000 and 5000 Decision Variables}

In the previous subsection, we have demonstrated the competitive performance of the proposed LMEA on benchmark MaOPs with the number of decision variables ranged from 100 to 1000 . In this subsection, we further challenge the proposed LMEA on MaOPs with larger scales.

Table VI presents the HV metric values obtained by the proposed LMEA on DTLZ1 to DTLZ7, WFG3, UF9 and UF10 with 2000 and 5000 decision variables, via 20 runs, showing that the proposed LMEA obtained a similar HV metric value on each test problem with both 2000 and 5000 variables. For further observations, Fig. 5 plots the IGD metric values of LMEA on 5-objective DTLZ3, 10-objective DTLZ6 and UF9 with different number of decision variables averaged over 20 runs. As evidenced in Fig. 5, the IGD metric values obtained by LMEA shows little deterioration on the three test problems as the number of decision variables increases from 100 to 5000, which indicates a promising scalability of the algorithm. 
TABLE VI

HV Metric VALues of LMEA ON DTLZ1 to DTLZ7, WFG3, UF9 AND UF10 WITH 2000 AND 5000 DeCISION VARIABLES, AVERAGING OVER 20 RUNS.

\begin{tabular}{cccc}
\hline Problem & Obj. & 2000-variable & 5000-variable \\
\hline \multirow{2}{*}{ DTLZ1 } & 5 & $9.3601 \mathrm{e}-1(6.41 \mathrm{e}-3)$ & $9.3782 \mathrm{e}-1(4.81 \mathrm{e}-3)$ \\
\cline { 2 - 4 } & 10 & $8.9433 \mathrm{e}-1(1.38 \mathrm{e}-2)$ & $8.7102 \mathrm{e}-1(1.31 \mathrm{e}-2)$ \\
\hline \multirow{2}{*}{ DTLZ2 } & 5 & $6.1363 \mathrm{e}-1(5.08 \mathrm{e}-3)$ & $6.1221 \mathrm{e}-1(4.75 \mathrm{e}-3)$ \\
\cline { 2 - 4 } & 10 & $6.4035 \mathrm{e}-1(2.19 \mathrm{e}-2)$ & $6.5006 \mathrm{e}-1(1.41 \mathrm{e}-2)$ \\
\hline \multirow{2}{*}{ DTLZ3 } & 5 & $6.1373 \mathrm{e}-1(4.85 \mathrm{e}-3)$ & $6.1270 \mathrm{e}-1(1.33 \mathrm{e}-2)$ \\
\cline { 2 - 4 } & 10 & $3.0131 \mathrm{e}-1(4.37 \mathrm{e}-2)$ & $1.5543 \mathrm{e}-1(2.88 \mathrm{e}-2)$ \\
\hline \multirow{2}{*}{ DTLZ4 } & 5 & $5.8063 \mathrm{e}-1(4.39 \mathrm{e}-2)$ & $5.9056 \mathrm{e}-1(2.04 \mathrm{e}-2)$ \\
\cline { 2 - 4 } & 10 & $7.3539 \mathrm{e}-1(3.52 \mathrm{e}-2)$ & $6.6224 \mathrm{e}-1(3.64 \mathrm{e}-2)$ \\
\hline \multirow{2}{*}{ DTLZ5 } & 5 & $3.4370 \mathrm{e}-2(2.75 \mathrm{e}-4)$ & $3.4090 \mathrm{e}-2(3.37 \mathrm{e}-4)$ \\
\cline { 2 - 4 } & 10 & $8.3512 \mathrm{e}-3(2.66 \mathrm{e}-4)$ & $7.8917 \mathrm{e}-3(5.19 \mathrm{e}-4)$ \\
\hline \multirow{2}{*}{ DTLZ6 } & 5 & $3.3410 \mathrm{e}-2(1.22 \mathrm{e}-3)$ & $3.4350 \mathrm{e}-2(1.10 \mathrm{e}-4)$ \\
\cline { 2 - 4 } & 10 & $6.6238 \mathrm{e}-3(1.49 \mathrm{e}-3)$ & $6.2831 \mathrm{e}-3(1.15 \mathrm{e}-3)$ \\
\hline \multirow{2}{*}{ DTLZ7 } & 5 & $1.2758 \mathrm{e}-1(4.62 \mathrm{e}-3)$ & $1.3263 \mathrm{e}-1(2.81 \mathrm{e}-3)$ \\
\cline { 2 - 4 } & 10 & $5.5823 \mathrm{e}-2(4.15 \mathrm{e}-3)$ & $5.7065 \mathrm{e}-2(7.94 \mathrm{e}-3)$ \\
\hline \multirow{2}{*}{ WFG3 } & 5 & $5.8731 \mathrm{e}-1(2.03 \mathrm{e}-3)$ & $5.8971 \mathrm{e}-1(3.06 \mathrm{e}-3)$ \\
\cline { 2 - 4 } & 10 & $6.0799 \mathrm{e}-1(2.75 \mathrm{e}-3)$ & $5.8697 \mathrm{e}-1(2.14 \mathrm{e}-3)$ \\
\hline UF9 & 3 & $7.0473 \mathrm{e}-1(1.29 \mathrm{e}-2)$ & $7.1139 \mathrm{e}-1(1.05 \mathrm{e}-2)$ \\
\hline UF10 & 3 & $1.7095 \mathrm{e}-1(7.70 \mathrm{e}-3)$ & $1.8247 \mathrm{e}-1(1.40 \mathrm{e}-2)$ \\
\hline
\end{tabular}

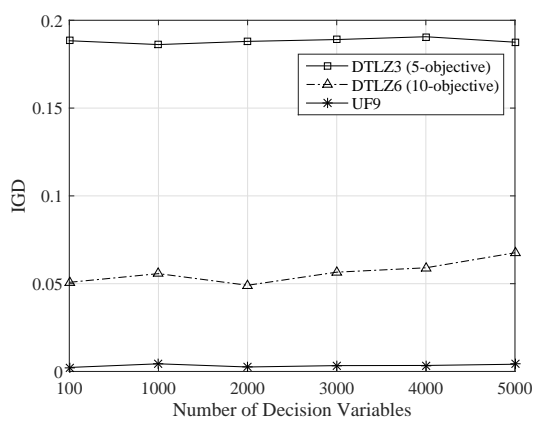

Fig. 5. IGD metric values of LMEA on 5-objective DTLZ3, 10objective DTLZ6 and UF9 with different numbers of decision variables, averaging over 20 runs.

\section{Performance Comparisons Between LMEA and Existing MOEAs on LSMOP Test Suit}

To further assess the performance of LMEA on more challenging large-scale MaOPs, more empirical exeperiments are conducted on a recently developed test suite for large-scale multi-objective and many-objective optimization, known as the LSMOP test suite [85]. The LSMOP test suite consists of nine test problems (i.e. LSMOP1-LSMOP9), which are constructed based on six widely used large-scale single-objective problems, namely, Sphere function, Schwefel's problem, Rosenbrock's function, Rastrigin's function, Griewank's function and Ackley's function. This class of test problems have the following main characteristics: 1) complicated variable linkages on the Pareto sets, 2) mixed separabilities between decision variables, 3) non-uniform non-separable variable groups, 4) non-uniform correlations between decision variables and objective functions, some of which are borrowed from the literature of large-scale single- objective optimization. Hence, LSMOP test problems can better reflect the challenges in large-scale MaOPs than existing test suits.

Table VII presents the statistical results of the IGD values obtained by the five algorithms on LSMOP1 to LSMOP9 with 5 objectives and 500 decision variables via 20 independent runs, where the parameter $T$ for controlling the ratio of knee points in KnEA is set to 0.5 , and all the other parameters are set to the same as in Section IV-A. It can be seen that the proposed LMEA is still able to achieve a competitive performance on the LSMOP test problems, which confirms the effectiveness of LMEA in solving challenging large-scale MaOPs.

\section{CONCLUSIONS AND REMARKS}

In this work, a decision variable clustering based evolutionary algorithm, termed LMEA, has been suggested for solving large-scale MaOPs. In LMEA, a decision variable clustering method has been developed to divide the decision variables into two groups, namely convergencerelated variables and diversity-related variables, according to their contributions to the convergence and diversity, respectively. Based on the grouping results, the two groups of variables are optimized separately. Moreover, a fast non-dominated sorting approach with a time complexity of $O(M N \log N / \log M)$, called T-ENS, has been proposed to improve the computational efficiency of LMEA, where $M$ is the number of objectives and $N$ is the size of population to perform non-dominated sorting. Empirical results on a variety of large-scale benchmark MaOPs that have up to 5000 decision variables demonstrate the promising scalability of LMEA in comparison with several sate-of-the-art MOEAs.

It has been shown that decision variable clustering is effective for solving large-scale MaOPs. While the proposed clustering method in this work only considers the decision variables to be convergence-related and diversity-related, in the future, we would like to investigate if it is possible to make more classifications of the decision variables, because decision variables in the same group may still have different (strong or weak) correlations with the objectives even if they are only related to convergence or diversity. In addition, we would also like to further improve the computational efficiency of the decision variable interaction analysis procedure, which is the main computational cost of the proposed LMEA.

\section{REFERENCES}

[1] P. J. Fleming, R. C. Purshouse, and R. J. Lygoe, "Many-objective optimization: an engineering design perspective," in Proceedings of the Third International Conference on Evolutionary multi-criterion optimization, 2005, pp. 14-32.

[2] J. G. Herrero, A. Berlanga, and J. M. M. López, "Effective evolutionary algorithms for many-specifications attainment: application to air traffic control tracking filters," IEEE Transactions on Evolutionary Computation, vol. 13, no. 1, pp. 151-168, 2009. 
TABLE VII

IGD Metric Values of the Five Algorithms on LSMOP1-LSMOP9, Where the Best Result on Each Test Instance is ShOWN in a GRAY BACKGROUND.

\begin{tabular}{cccccc}
\hline Problem & MOEA/D & NSGA-III & KnEA & MOEA/DVA & LMEA \\
\hline LSMOP1 & $3.6215 \mathrm{e}-1(2.77 \mathrm{e}-2)-$ & $2.0411 \mathrm{e}-1(3.05 \mathrm{e}-3)-$ & $6.5295 \mathrm{e}-1(3.88 \mathrm{e}-1)-$ & $1.7219 \mathrm{e}-1(7.47 \mathrm{e}-3)-$ & $1.5151 \mathrm{e}-1(9.99 \mathrm{e}-3)$ \\
LSMOP2 & $2.4541 \mathrm{e}-1(8.98 \mathrm{e}-4)-$ & $1.4727 \mathrm{e}-1(1.67 \mathrm{e}-3)-$ & $2.3724 \mathrm{e}-1(7.06 \mathrm{e}-2)-$ & $1.4212 \mathrm{e}-1(2.16 \mathrm{e}-3)-$ & $1.2644 \mathrm{e}-1(1.45 \mathrm{e}-3)$ \\
LSMOP3 & $7.0884 \mathrm{e}-1(4.00 \mathrm{e}-2)-$ & $4.4176 \mathrm{e}-1(1.30 \mathrm{e}-1) \approx$ & $7.0241 \mathrm{e}-1(9.37 \mathrm{e}-2)-$ & $6.9024 \mathrm{e}-1(4.55 \mathrm{e}-2)-$ & $4.1242 \mathrm{e}-1(4.68 \mathrm{e}-2)$ \\
LSMOP4 & $2.7326 \mathrm{e}-1(4.55 \mathrm{e}-3)-$ & $1.8222 \mathrm{e}-1(9.13 \mathrm{e}-3)-$ & $6.0172 \mathrm{e}-1(1.44 \mathrm{e}-1)-$ & $1.5548 \mathrm{e}-1(4.40 \mathrm{e}-3) \approx$ & $1.5585 \mathrm{e}-1(2.04 \mathrm{e}-3)$ \\
LSMOP5 & $5.7900 \mathrm{e}-1(6.32 \mathrm{e}-2)-$ & $3.2983 \mathrm{e}-1(1.23 \mathrm{e}-1)-$ & $1.1584 \mathrm{e}+0(4.06 \mathrm{e}-1)-$ & $3.8667 \mathrm{e}-1(3.92 \mathrm{e}-2)-$ & $2.6932 \mathrm{e}-1(1.65 \mathrm{e}-2)$ \\
LSMOP6 & $1.2119 \mathrm{e}+0(2.44 \mathrm{e}-1)+$ & $1.1094 \mathrm{e}+0(1.22 \mathrm{e}-1)+$ & $1.8486 \mathrm{e}+0(1.39 \mathrm{e}+0)+$ & $2.0992 \mathrm{e}+0(1.79 \mathrm{e}-1)+$ & $1.3820 \mathrm{e}+4(3.91 \mathrm{e}+3)$ \\
LSMOP7 & $9.9083 \mathrm{e}-1(1.53 \mathrm{e}-1)+$ & $1.0033 \mathrm{e}+0(2.11 \mathrm{e}-1)+$ & $1.2275 \mathrm{e}+1(9.00 \mathrm{e}+0)-$ & $9.1631 \mathrm{e}-1(2.81 \mathrm{e}-2)+$ & $1.3542 \mathrm{e}+0(3.00 \mathrm{e}-1)$ \\
LSMOP8 & $5.1604 \mathrm{e}-1(1.67 \mathrm{e}-2)-$ & $2.9337 \mathrm{e}-1(1.02 \mathrm{e}-2)-$ & $4.3832 \mathrm{e}-1(5.81 \mathrm{e}-3)-$ & $3.0537 \mathrm{e}-1(6.19 \mathrm{e}-2)-$ & $2.3022 \mathrm{e}-1(7.57 \mathrm{e}-3)$ \\
LSMOP9 & $1.0600 \mathrm{e}+0(2.31 \mathrm{e}-1)-$ & $2.3568 \mathrm{e}+0(8.52 \mathrm{e}-2)-$ & $1.3535 \mathrm{e}+0(6.62 \mathrm{e}-1) \approx$ & $6.5805 \mathrm{e}-1(1.13 \mathrm{e}-1) \approx$ & $6.1163 \mathrm{e}-1(3.55 \mathrm{e}-2)$ \\
\hline
\end{tabular}

[3] J. B. Kollat, P. M. Reed, and R. Maxwell, “Many-objective groundwater monitoring network design using bias-aware ensemble kalman filtering, evolutionary optimization, and visual analytics," Water Resources Research, vol. 47, no. 2, pp. 1-18, 2011.

[4] J. W. Kruisselbrink, M. T. Emmerich, T. Bäck, A. Bender, A. P. IJzerman, and E. van der Horst, "Combining aggregation with Pareto optimization: a case study in evolutionary molecular design," in Proceedings of the fifth International Conference on Evolutionary MultiCriterion Optimization, 2009, pp. 453-467.

[5] K. Ikeda, H. Kita, and S. Kobayashi, "Failure of Pareto-based MOEAs: does non-dominated really mean near to optimal?" in Proceedings of the 2001 IEEE Congress on Evolutionary Computation, 2001, pp. 957-962.

[6] V. Khare, X. Yao, and K. Deb, "Performance scaling of multiobjective evolutionary algorithms," in Proceedings of the Second International Conference on Evolutionary Multi-Criterion Optimization, 2003, pp. 376-390.

[7] D. Brockhoff, T. Friedrich, N. Hebbinghaus, C. Klein, F. Neumann, and E. Zitzler, "On the effects of adding objectives to plateau functions," IEEE Transactions on Evolutionary Computation, vol. 13, no. 3, pp. 591-603, 2009.

[8] R. C. Purshouse and P. J. Fleming, "Evolutionary many-objective optimization: an exploratory analysis," in Proceedings of 2003 Congress on Evolutionary Computation, 2003, pp. 2066-2073.

[9] R. C. Purshouse and P. J. Fleming(2007), "On the evolutionary optimization of many conflicting objectives," IEEE Transactions on Evolutionary Computation, vol. 11, no. 6, pp. 770-784, 2007.

[10] P. J. Fleming, R. C. Purshouse, and R. J. Lygoe, "Many-objective optimization: an engineering design perspective," in Proceedings of the International Conference on Evolutionary multi-criterion optimization, 2005, pp. 14-32.

[11] S. Chand and M. Wagner, "Evolutionary many-objective optimization: a quick-start guide," Surveys in Operations Research and Management Science, vol. 20, no. 2, pp. 35-42, 2015.

[12] B. Li, J. Li, K. Tang, and X. Yao, "Many-objective evolutionary algorithms: a survey," ACM Computing Surveys, vol. 48, no. 1, pp. 13:1-13:37, 2015

[13] M. Laumanns, L. Thiele, K. Deb, and E. Zitzler, "Combining convergence and diversity in evolutionary multiobjective optimization," Evolutionary Computation, vol. 10, no. 3, pp. 263-282, 2002.

[14] D. Hadka and P. Reed, "Borg: an auto-adaptive many-objective evolutionary computing framework," Evolutionary Computation, vol. 21, no. 2, pp. 231-259, 2013

[15] X. Zou, Y. Chen, M. Liu, and L. Kang, "A new evolutionary algorithm for solving many-objective optimization problems," IEEE Transactions on Systems, Man, and Cybernetics-Part B, vol. 38, no. 5, pp. 1402-1412, 2008.

[16] G. Wang and H. Jiang, "Fuzzy-dominance and its application in evolutionary many objective optimization," in Proceedings of the 2007 International Conference on Computational Intelligence and Security Workshops, 2007, pp. 195-198.

[17] F. di Pierro, S.-T. Khu, and D. A. Savić, "An investigation on preference order ranking scheme for multiobjective evolutionary optimization," IEEE Transactions on Evolutionary Computation, vol. 11, no. 1, pp. 17-45, 2007.

[18] Y. Yuan, H. Xu, B. Wang, and X. Yao, "A new dominance relation based evolutionary algorithm for many-objective optimization," IEEE Transactions on Evolutionary Computation, vol. 20, no. 1, pp. $16-37,2015$.

[19] M. Köppen and K. Yoshida, "Substitute distance assignments in NSGA-II for handling many-objective optimization problems," in Proceedings of the 4th International Conference on Evolutionary MultiCriterion Optimization, 2007, pp. 727-741.

[20] S. Yang, M. Li, X. Liu, and J. Zheng, "A grid-based evolutionary algorithm for many-objective optimization," IEEE Transactions on Evolutionary Computation, vol. 17, no. 5, pp. 721-736, 2013.

[21] R. Wang, R. C. Purshouse, and P. J. Fleming, "Preferenceinspired coevolutionary algorithms for many-objective optimization," IEEE Transactions on Evolutionary Computation, vol. 17, no. 4, pp. 474-494, 2013.

[22] J. Cheng, G. Yen, and G. Zhang, "A many-objective evolutionary algorithm with enhanced mating and environmental selections," IEEE Transactions on Evolutionary Computation, vol. 19, pp. 592-605, 2015.

[23] X. Zhang, Y. Tian, and Y. Jin, "A knee point driven evolutionary algorithm for many-objective optimization," IEEE Transactions on Evolutionary Computation, vol. 19, no. 6, pp. 761-776, 2014.

[24] C. A. Rodríguez Villalobos and C. A. Coello Coello, "A new multi-objective evolutionary algorithm based on a performance assessment indicator," in Proceedings of the 14th Annual Conference on Genetic and Evolutionary Computation, 2012, pp. 505-512.

[25] T. Ulrich, J. Bader, and L. Thiele, "Defining and optimizing indicator-based diversity measures in multiobjective search," in Proceedings of the 11th International Conference on Parallel Problem Solving from Nature, 2010, pp. 707-717.

[26] H. Trautmann, T. Wagner, and D. Brockhoff, "R2-EMOA: focused multiobjective search using R2-indicator-based selection," in Proceedings of the 7th International Conference on Learning and Intelligent Optimization, 2013, pp. 70-74.

[27] G. Rudolph, O. Schütze, C. Grimme, and H. Trautmann, "An aspiration set EMOA based on averaged hausdorff distances," in Proceedings of the 8th International Conference on Learning and Intelligent Optimization, 2014, pp. 153-156.

[28] E. Zitzler and S. Künzli, "Indicator-based selection in multiobjective search," in Proceedings of the 8th International Conference on Parallel Problem Solving from Nature, 2004, pp. 832-842.

[29] N. Beume, B. Naujoks, and M. Emmerich, "SMS-EMOA: multiobjective selection based on dominated hypervolume," European Journal of Operational Research, vol. 181, no. 3, pp. 1653-1669, 2007. 
[30] J. Bader and E. Zitzler, "HypE: an algorithm for fast hypervolumebased many-objective optimization," Evolutionary Computation, vol. 19, no. 1, pp. 45-76, 2011.

[31] Q. Zhang and H. Li, "MOEA/D: a multi-objective evolutionary algorithm based on decomposition," IEEE Transactions on Evolutionary Computation, vol. 11, no. 6, pp. 712-731, 2007.

[32] K. Li, Q. Zhang, S. Kwong, M. Li, and R. Wang, "Stable matchingbased selection in evolutionary multiobjective optimization," IEEE Transactions on Evolutionary Computation, vol. 18, no. 6, pp. 909-923, 2014.

[33] Y. Qi, X. Ma, F. Liu, L. Jiao, J. Sun, and J. Wu, "MOEA/D with adaptive weight adjustment," Evolutionary computation, vol. 22, no. 2, pp. 231-264, 2014.

[34] X. Cai, Y. Li, Z. Fan, and Q. Zhang, "An external archive guided multiobjective evolutionary algorithm based on decomposition for combinatorial optimization," IEEE Transactions on Evolutionary Computation, vol. 19, no. 4, pp. 508-523, 2015.

[35] Y. Yuan, H. Xu, B. Wang, B. Zhang, and X. Yao, "Balancing convergence and diversity in decomposition-based many-objective optimizers," IEEE Transactions on Evolutionary Computation, vol. 20, no. 2, pp. 180-198, 2016.

[36] S. B. Gee, K. C. Tan, V. A. Shim, and N. R. Pal, "Online diversity assessment in evolutionary multiobjective optimization: a geometrical perspective," IEEE Transactions on Evolutionary Computation, vol. 19, no. 4, pp. 542-559, 2015.

[37] H.-L. Liu, F. Gu, and Q. Zhang, "Decomposition of a multiobjective optimization problem into a number of simple multiobjective subproblems," IEEE Transactions on Evolutionary Computation, vol. 18, no. 3, pp. 450-455, 2014.

[38] K. Deb and H. Jain, "An evolutionary many-objective optimization algorithm using reference-point based non-dominated sorting approach, part I: solving problems with box constraints," IEEE Transactions on Evolutionary Computation, vol. 18, no. 4, pp. 577601, 2014

[39] K. Li, K. Deb, Q. Zhang, and S. Kwong, "Combining dominance and decomposition in evolutionary many-objective optimization," IEEE Transactions on Evolutionary Computation, vol. 19, no. 5, pp. 694-716, 2015.

[40] R. Cheng, Y. Jin, M. Olhofer, and B. Sendhoff, "A reference vector guided evolutionary algorithm for many-objective optimization," IEEE Transactions on Evolutionary Computation, 2016, in press.

[41] D. Brockhoff and E. Zitzler, "Are all objectives necessary? on dimensionality reduction in evolutionary multiobjective optimization," in Proceedings of 9th International Conference on Parallel Problem Solving from Nature, 2006, pp. 533-542.

[42] D. Brockhoff and E. Zitzler(2009), "Objective reduction in evolutionary multiobjective optimization: theory and applications," Evolutionary Computation, vol. 17, no. 2, pp. 135-166, 2009.

[43] A. López Jaimes, C. A. Coello Coello, and D. Chakraborty, "Objective reduction using a feature selection technique," in Proceedings of the 10th Annual Conference on Genetic and Evolutionary Computation, 2008, pp. 673-680.

[44] H. K. Singh, A. Isaacs, and T. Ray, "A Pareto corner search evolutionary algorithm and dimensionality reduction in manyobjective optimization problems," IEEE Transactions on Evolutionary Computation, vol. 15, no. 4, pp. 539-556, 2011.

[45] K. Deb and D. Saxena, "Searching for Pareto-optimal solutions through dimensionality reduction for certain large-dimensional multi-objective optimization problems," in Proceedings of the World Congress on Computational Intelligence, 2006, pp. 3352-3360.

[46] D. K. Saxena and K. Deb, "Non-linear dimensionality reduction procedures for certain large-dimensional multi-objective optimization problems: employing correntropy and a novel maximum variance unfolding," in Proceedings of 4th International Conference on Evolutionary Multi-Criterion Optimization, 2007, pp. 772-787.

[47] D. K. Saxena, J. A. Duro, A. Tiwari, K. Deb, and Q. Zhang, "Objective reduction in many-objective optimization: linear and nonlinear algorithms," IEEE Transactions on Evolutionary Computation, vol. 17, no. 1, pp. 77-99, 2013.

[48] H. Wang and X. Yao, "Objective reduction based on nonlinear correlation information entropy," Soft Computing, pp. 1-15, 2015.

[49] M. Li, S. Yang, and X. Liu, "Bi-goal evolution for many-objective optimization problems," Artificial Intelligence, vol. 228, pp. 45-65, 2015.

[50] B.-Y. Qu and P. N. Suganthan, "Multi-objective evolutionary algorithms based on the summation of normalized objectives and diversified selection," Information sciences, vol. 180, no. 17, pp. 3170-3181, 2010.

[51] K. Bringmann, T. Friedrich, C. Igel, and T. Voß, "Speeding up many-objective optimization by Monte Carlo approximations," Artificial Intelligence, vol. 204, pp. 22-29, 2013.

[52] K. M. Clymont and E. Keedwell, "Deductive sort and climbing sort: new methods for non-dominated sorting," Evolutionary Computation, vol. 20, no. 1, pp. 1-26, 2012.

[53] H. Wang and X. Yao, "Corner sort for Pareto-based manyobjective optimization," IEEE Transactions on Cybernetics, vol. 44, no. 1, pp. 92-102, 2014.

[54] M. Drozdík, Y. Akimoto, H. Aguirre, and K. Tanaka, "Computational cost reduction of non-dominated sorting using M-front," IEEE Transactions on Evolutionary Computation, vol. 19, no. 5, pp. 659-678, 2015.

[55] X. Zhang, Y. Tian, R. Cheng, and Y. Jin, "An efficient approach to non-dominated sorting for evolutionary multi-objective optimization," IEEE Transactions on Evolutionary Computation, vol. 19, no. 2, pp. 201-213, 2015.

[56] X. Zhang, Y. Tian, and Y. Jin, "Approximate non-dominated sorting for evolutionary many-objective optimization," Information Sciences, 2016, in press.

[57] Z. Yang, K. Tang, and X. Yao, "Large scale evolutionary optimization using cooperative coevolution," Information Sciences, vol. 178, no. 15, pp. 2985-2999, 2008.

[58] M. N. Omidvar, X. Li, Y. Mei, and X. Yao, "Cooperative coevolution with differential grouping for large scale optimization," IEEE Transactions on Evolutionary Computation, vol. 18, no. 3, pp. 378-393, 2014

[59] X. Li and X. Yao, "Cooperatively coevolving particle swarms for large scale optimization," IEEE Transactions on Evolutionary Computation, vol. 16, no. 2, pp. 210-224, 2012.

[60] R. Cheng and Y. Jin, "A competitive swarm optimizer for large scale optimization," IEEE Transactions on Cybernetics, vol. 45, no. 2 , pp. 191-204, 2015.

[61] K. H. Hajikolaei, Z. Pirmoradi, G. H. Cheng, and G. G. Wang, "Decomposition for large-scale global optimization based on quantified variable correlations uncovered by metamodelling," Engineering Optimization, vol. 47, no. 4, pp. 429-452, 2015.

[62] J. Fan, J. Wang, and M. Han, “Cooperative coevolution for largescale optimization based on kernel fuzzy clustering and variable trust region methods," IEEE Transactions on Fuzzy Systems, vol. 22, no. 4, pp. 829-839, 2014.

[63] R. Cheng and Y. Jin, "A social learning particle swarm optimization algorithm for scalable optimization," Information Sciences, vol. 291, pp. 43-60, 2015.

[64] L. M. Antonio and C. A. Coello Coello, "Use of cooperative coevolution for solving large scale multiobjective optimization problems," in Proceedings of 2013 IEEE Congress on Evolutionary Computation, 2013, pp. 2758-2765.

[65] X. Ma, F. Liu, Y. Qi, X. Wang, L. Li, L. Jiao, M. Yin, and M. Gong, "A multiobjective evolutionary algorithm based on decision variable analyses for multi-objective optimization problems with large scale variables," IEEE Transactions on Evolutionary Computation, vol. 20, no. 2, pp. 275-298, 2016.

[66] R. Shang, K. Dai, L. Jiao, and R. Stolkin, "Improved memetic algorithm based on route distance grouping for multiobjective large scale capacitated arc routing problems," IEEE Transactions on Cybernetics, vol. 46, no. 4, pp. 1000-1013, 2016.

[67] W. Chen, T. Weise, Z. Yang, and K. Tang, "Large-scale global optimization using cooperative coevolution with variable interaction learning," in Proceedings of 11th International Conference on Parallel Problem Solving from Nature, 2010, pp. 300-309.

[68] K. Deb and R. B. Agrawal, "Simulated binary crossover for continuous search space," Complex Systems, vol. 9, no. 4, pp. 115148, 1995.

[69] K. Deb and M. Goyal, "A combined genetic adaptive search (GeneAS) for engineering design," Computer Science and Informatics, vol. 26, no. 4, pp. 30-45, 1996.

[70] B. Babu and M. Jehan, "Differential evolution for multi-objective optimization," in Proceedings of 2003 Congress on Evolutionary Computation, vol. 4, 2003, pp. 2696-2703.

[71] T. Robič and B. Filipič, "DEMO: differential evolution for multiobjective optimization," in Proceedings of Third International Conference on Evolutionary Multi-Criterion Optimization, 2005, pp. 520533. 
[72] K. Deb, A. Pratap, S. Agarwal, and T. Meyarivan, "A fast and elitist multi-objective genetic algorithm: NSGA-II," IEEE Transactions on Evolutionary Computation, vol. 6, no. 2, pp. 182-197, 2002.

[73] O. Schütze, "A new data structure for the nondominance problem in multi-objective optimization," in Proceedings of the 2nd International Conference on Evolutionary Multi-Criterion Optimization, 2003, pp. 509-518.

[74] H. Fang, Q. Wang, Y. Tu, and M. F. Horstemeyer, "An efficient non-dominated sorting method for evolutionary algorithms," Evolutionary Computation, vol. 16, no. 3, pp. 355-384, 2008.

[75] K. Deb, L. Thiele, M. Laumanns, and E. Zitzler, Scalable test problems for evolutionary multiobjective optimization. Springer, 2005.

[76] L. B. S. Huband, P. Hingston and L. While, "A review of multiobjective test problems and a scalable test problem toolkit," IEEE Transactions on Evolutionary Computation, vol. 10, no. 5, pp. 477506, 2006.

[77] Q. Zhang, A. Zhou, S. Zhao, P. N. Suganthan, W. Liu, and S. Tiwari, "Multiobjective optimization test instances for the CEC 2009 special session and competition," University of Essex, Colchester, UK and Nanyang technological University, Singapore, special session on performance assessment of multi-objective optimization algorithms, technical report, pp. 1-30, 2008.

[78] H. Li and Q. Zhang, "Multiobjective optimization problems with complicated Pareto sets, MOEA/D and NSGA-II," IEEE Transactions on Evolutionary Computation, vol. 13, no. 2, pp. 284-302, 2009.

[79] A. Zhou, Y. Jin, Q. Zhang, B. Sendhoff, and E. Tsang, "Combining model-based and genetics-based offspring generation for multiobjective optimization using a convergence criterion," in Proceedings of 2006 IEEE Congress on Evolutionary Computation, 2006, pp. 892-899.

[80] L. While, P. Hingston, L. Barone, and S. Huband, "A faster algorithm for calculating hypervolume," IEEE Transactions on Evolutionary Computation, vol. 10, no. 1, pp. 29-38, 2006.

[81] L. Russo and A. P. Francisco, "Quick hypervolume," IEEE Transactions on Evolutionary Computation, vol. 18, no. 4, pp. 481-502, 2014.

[82] I. Das and J. E. Dennis, "Normal-boundary intersection: A new method for generating the pareto surface in nonlinear multicriteria optimization problems," SIAM Journal on Optimization, vol. 8, no. 3, pp. 631-657, 1998.

[83] R. Cheng, Y. Jin, and K. Narukawa, "Adaptive reference vector generation for inverse model based evolutionary multiobjective optimization with degenerate and disconnected Pareto fronts," in Proceedings of the Evolutionary Multi-Criterion Optimization, 2015, pp. 127-140.

[84] L.-Y. Tseng and C. Chen, "Multiple trajectory search for unconstrained/constrained multi-objective optimization," in Proceedings of 2009 IEEE Congress onEvolutionary Computation, 2009, pp. 19511958.

[85] R. Cheng, Y. Jin, M. Olhofer, and B. Sendhoff, "Test problems for large-scale multi- and many-objective optimization," IEEE Transactions on Cybernetics, 2016, in press.



Xingyi Zhang received the B.Sc. from Fuyang Normal College in 2003, and the M.Sc. in 2006 and Ph.D. in 2009 both from Huazhong University of Science and Technology. Currently, he is a professor in the School of Computer Science and Technology, Anhui University. His main research interests include unconventional models and algorithms of computation, multi-objective optimization and membrane computing.

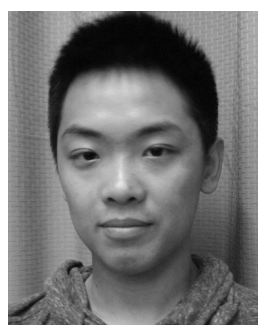

Ye Tian received the B.Sc. in 2012, and the M.Sc. in 2015 both from Anhui University. Currently, he is a Ph.D. degree candidate in the School of Computer Science and Technology, Anhui University. His current research interest is multiobjective optimization methods and their application.

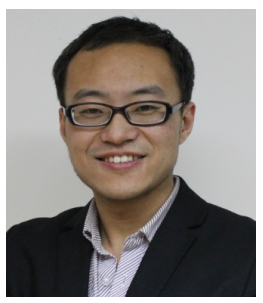

Ran Cheng received the B. Eng. degree from the Northeastern University, Shenyang, China, in 2010, and Ph.D. degree from the University of Surrey, United Kingdom, in 2016. He is currently a research fellow with the School of Computer Science, University of Birmingham, United Kingdom. His research interests include multi-/many-objective optimization, large-scale optimization, model based evolutionary algorithms, and swarm intelligence.



Yaochu Jin (M'98-SM'02) received the B.Sc., M.Sc., and Ph.D. degrees from Zhejiang University, Hangzhou, China, in 1988, 1991, and 1996 respectively, and the Dr.-Ing. degree from Ruhr University Bochum, Germany, in 2001.

$\mathrm{He}$ is a Professor in Computational Intelligence, Department of Computer Science, University of Surrey, Guildford, U.K., where he heads the Nature Inspired Computing and Engineering Group. He is also a Finland Distinguished Professor funded by the Finnish Agency for Innovation (Tekes) and a Changjiang Distinguished Visiting Professor appointed by the Ministry of Education, China. His sciencedriven research interests lie in the interdisciplinary areas that bridge the gap between computational intelligence, computational neuroscience, and computational systems biology. $\mathrm{He}$ is also particularly interested in nature-inspired, real-world driven problem-solving. He has (co)authored over 200 peer-reviewed journal and conference papers and been granted eight patents on evolutionary optimization. His current research is funded by EC FP7, UK EPSRC and industry. He has delivered 20 invited keynote speeches at international conferences.

He is Editor-in-Chief of the IEEE TRANSACTIONS ON COGNITIVE AND DEVELOPMENTAL SYSTEMS and Complex \& Intelligent Systems. He is also an Associate Editor or Editorial Board Member of the IEEE TRANSACTIONS ON EVOLUTIONARY COMPUTATION, IEEE TRANSACTIONS ON CYBERNETICS, IEEE TRANSACTIONS ON NANOBIOSCIENCE, Evolutionary Computation, BioSystems, Soft Computing, and Natural Computing. Dr Jin was an IEEE Distinguished Lecturer (2013-2015) and Vice President for Technical Activities of the IEEE Computational Intelligence Society (2014-2015). He was the recipient of the Best Paper Award of the 2010 IEEE Symposium on Computational Intelligence in Bioinformatics and Computational Biology and the 2014 IEEE Computational Intelligence Magazine Outstanding Paper Award. He is a Fellow of IEEE. 